\title{
Algal assemblages in springs of different lithologies (ophiolites vs. limestone) of the Konjuh Mountain (Bosnia and Herzegovina)
}

\author{
Jasmina Kamberovići ${ }^{1 \star}$, Anđelka Plenković-Moraj², Koraljka Kralj Borojević3 ${ }^{3}$, Marija Gligora \\ Udovič², Petar Žutinić ${ }^{2}$, Dubravka Hafner ${ }^{4}$, Marco Cantonati ${ }^{5}$ \\ ${ }^{1}$ University of Tuzla, Faculty of Natural Sciences and Mathematics, Univerzitetska 4, 75000, Tuzla, Bosnia and Herze- \\ govina, jasmina.kamberovic@untz.ba \\ ${ }^{2}$ University of Zagreb, Faculty of Science, Department of Biology, Rooseveltov trg 6, 10000 Zagreb, Croatia \\ ${ }^{3}$ Minnesota Drive, Great Sankey, Warrington, UK, WA5 3SY \\ ${ }^{4}$ Bartulovići 4, 20357 Blace, Croatia \\ ${ }^{5}$ MUSE - Museo delle Scienze, Limnology and Phycology Section, Corso del Lavoro e della Scienza 3, I-38123 Trento, \\ Italy
}

\begin{abstract}
The biodiversity of algal communities and environmental conditions were investigated in the springs of Mt. Konjuh. The assemblages of 20 springs emerging from different lithologies (limestones and ophiolites, respectively) comprised 234 algal taxa. Diatoms and cyanobacteria were the most species-rich groups. The most common alkaliphilic, circumneutral, and eutraphentic diatoms were represented by the genera Gomphonema, Nitzschia, Navicula, Cymbella, and Achnanthidium, and by the cyanobacterial genus Phormidium. Hierarchical clustering and SIMPROF analysis based on relative algal abundance clustered springs into six groups, separating them mainly according to spring type and lithology. Indicator species for groups and springs on different lithological substrata were singled out, revealing 33 taxa with preferences for ophiolites, and 20 taxa with preferences for carbonates. The values of the Shannon-Wiener diversity index were moderately high per spring location, and similar for the two groups of springs on different lithologies. A higher similarity in species composition was noted between springs on ophiolites and limestones than between springs on ophiolites and other types of siliceous substrata. The present study suggests that algal assemblages in springs emerging from ophiolites, even those made up by a preponderance of silicates, should be analyzed separately from those related to springs on other siliceous substrata. The results obtained showed that most of the springs studied are affected by anthropogenic impacts and morphological alterations leading to the dominance of highly competitive meso- and eutraphentic algal species, thus emphasizing the importance of further investigation and conservation of these habitats.
\end{abstract}

Keywords: biodiversity, diatoms, cyanobacteria, springs, geological substratum, ecological preferences, trophic status

\section{Introduction}

The importance of spring habitats as biodiversity hotspots and refugia for biodiversity conservation (Cantonati et al. 2012a, Taxböck et al. 2017) has been emphasized by the description of a large number of rare and endangered algal species (Sabater and Roca 1990, 1992, Cantonati 1998, Werum 2001, Poulíčková et al. 2005, Nascimbene et al. 2011). Half of the pennate freshwater diatoms described in Europe were recorded from spring ecosystems (Werum and Lange Bertalot 2004). Studies dealing with the algal biodiversity of European springs have increased since the mid 1990s (Sabat- er and Roca 1990, Cantonati 1998, Falasco and Bona 2011, Werum and Lange-Bertalot 2004, Fránková et al. 2009). Some of them resulted in the discovery of new diatom genera and species (Cantonati and Lange-Bertalot 2006, 2009, 2010, 2011, Cantonati et al. 2009, 2010). Studies on algae in Bosnia and Herzegovina date back to the late $19^{\text {th }}$ century and the early $20^{\text {th }}$ century (Protić $1897,1899,1901,1908$, Gutwinski 1899, Beck 1928), whereas the first detailed studies on diatoms were carried out in the framework of a wider study of diatom assemblages in several lakes and springs of 
the Balkan Peninsula (Hustedt 1945). However, crenic algal assemblages in Bosnia and Herzegovina have been investigated only at a few karst spring locations (Blagojević 1974, 1976, 1979, Hafner 2009, Dedić et al. 2015).

Results deriving from the study of algal ecology in springs (Cantonati et al. 2012b, c) have application in the monitoring of freshwater ecosystems. As a commonly studied algal group, diatoms are a useful proxy for water quality, reflecting both physical and chemical characteristics of a water body (Kelly and Whitton 1995, Rott et al. 1999, Werum and Lange-Bertalot 2004, Wojtal 2013). Diatom assemblages can be grouped according to different lithology (Werum and Lange-Bertalot 2004). For instance, Cymbella tridentina Lange-Bertalot, M. Cantonati \& A. Scalfi is a typical crenophilous species living on carbonate substrate (Cantonati et al. 2010), while Achnanthidium dolomiticum M. Cantonati et Lange-Bertalot is more common in springs fed by drainage basins dominated by dolomite lithology (Cantonati and Lange-Bertalot 2006). Diatom genera such as Achnanthes s.l. and Cymbella are generally more represented on carbonate substrates, whilst Eunotia and Pinnularia are more frequent on silicate rocks (Cantonati 1998).

Algal assemblages in springs on ophiolitic or ultramafic rocks have been rarely studied (Werum 2001). Although widespread on several continents (Dilek and Furnes 2011), these substrata cover less than 1\% of the Earth's surface (Coleman and Jove 1992). Dinaric ophiolites constitute one of the largest exposures of mantle rocks on Earth (Lugović et al. 1991). Ophiolites from Bosnia belong to the western belt of ophiolites in the Balkans as fragments of a long chain which passes through western Serbia (Mt. Zlatibor), Kosovo and the western belt of the Mirdita ophiolite in Albania to the Pindos and Othris ophiolites in central Greece. The ophiolites and associated sediments (mostly carbonates and ophiolitic melange) cover a surface of tens of thousands square kilometers in Bosnia and Herzegovina, with the KrivajaKonjuh mountain complex, which is the largest ophiolite zone in this area, positioned in the north-eastern part of the country (Trubelja et al. 1995, Babajić 2009). Still, despite its wide-ranging coverage, freshwater algal assemblages on Dinaric ophiolite substrata are largely unexplored. Unlike other siliceous rocks, ophiolites have less than $45 \%$ concentrations of silica, generally contain high amounts of $\mathrm{Fe}$, and trace metals such as Ni and Cr (Alexander et al. 2007, Trubelja et al. 1995). Results of chemical studies on ultramaphic rocks of the Krivaja-Konjuh ophiolitic complex demonstrated modal composition with high content of $\mathrm{MgO}$, low content of $\mathrm{CaO}$, and a high $\mathrm{MgO}: \mathrm{FeO}$ ratio of about 5 and more (Operta 2017).

The main objectives of this study were to: (i) explore algal biodiversity in crenic habitats of Mt. Konjuh, north-eastern Bosnia and Herzegovina, including rarely investigated springs on ophiolites, (ii) describe ecomorphological, physical and chemical properties of these spring habitats, (iii) analyze the influence of geological substrata on algal assemblages, and (iv) describe the ecological preferences of dominant algal groups.

\section{Materials and methods}

\section{Study area}

Konjuh Mountain is a part of the central Dinarides on the Balkan Peninsula, with an altitude of $1326 \mathrm{~m}$ a.s.l. In 2009 it was designated a Protected Landscape (IUCN Category V) with ecosystems characterized as Tertiary relicts. It belongs to a zone with a moderately continental climate, with a mean annual temperature varying between $9.2-10{ }^{\circ} \mathrm{C}$, and annual precipitation ranging from 900 to $1250 \mathrm{~mm}$ (Kudumović Dostović 2012). As part of the ophiolitic complex, Mt. Konjuh consists mainly of peridotite and serpentinite rocks. In addition, ophiolitic melange, gabbro, shale and limestones are also found (Ristić et al. 1967).

\section{Physical and chemical analyses}

Ecomorphological characteristics of springs (shading, current velocity, spring type) were assessed using methods described in Spitale (2007). Discharge was measured by a graduated bucket. The flow discharge for large rheocrenic springs was estimated by multiplying the cross section area, obtained from the values of average depth and width of the stream channel, and average velocity, which was measured by the float method. Temperature, dissolved oxygen, $\mathrm{pH}$, and conductivity were measured directly on site with a Hanna HI 9828 Multimeter (Hanna Instruments Inc., U.S.A.). Water for analyses of chemical parameters was taken at the same time. Total alkalinity, calcium, and magnesium were analyzed via titration, and sulphates were measured using the turbidimetric method (APHA, AWWA and WEF, 1999). Ammonia, nitrates and nitrites were analyzed via a spectrometric method according to the standard procedures (BAS ISO 7150-1: 2002, BAS ISO 7890-3:2002, BAS EN 26777:2000) in Bosnia and Herzegovina (Institute for Standardization of Bosnia and Herzegovina, 2002a, b, 2000, respectively).

\section{Algal sampling and identification}

A total of 196 algal samples were collected at the springhead and 15 meters downstream during the spring, summer and autumn of 2013. Altogether 20 springs were seasonally studied: nine springs on ophiolites (2 rheocrenes, 6 rheohelocrenes, and one hygropetric spring), one (rheohelocrenic) spring on ophiolitic melange, and ten springs on carbonate (limestone) substrate (7 rheocrenes and 3 rheohelocrenes). The springs are situated at altitudes ranging between 402 and $1003 \mathrm{~m}$ a.s.l (Fig. 1). Epilithic algae were taken by brushing the surfaces of 5-6 stones (Kelly et al. 1998), samples of epibryon were collected from aquatic bryophytes, while epipelon was collected from the sandy bottom with a cylinder.

Abundance of diatom and non-diatom algae was estimated as number of cells per square centimeter (epilithon and epipelon) or number of cells per gram of dried bryophyte mass. Non-diatom species were identified in fresh samples, whilst diatoms were acid cleaned (Hustedt 1930) and mounted in Naphrax (Brunel Microscopes Ltd., U.K.). At least 400 diatom valves were identified in each slide using random 


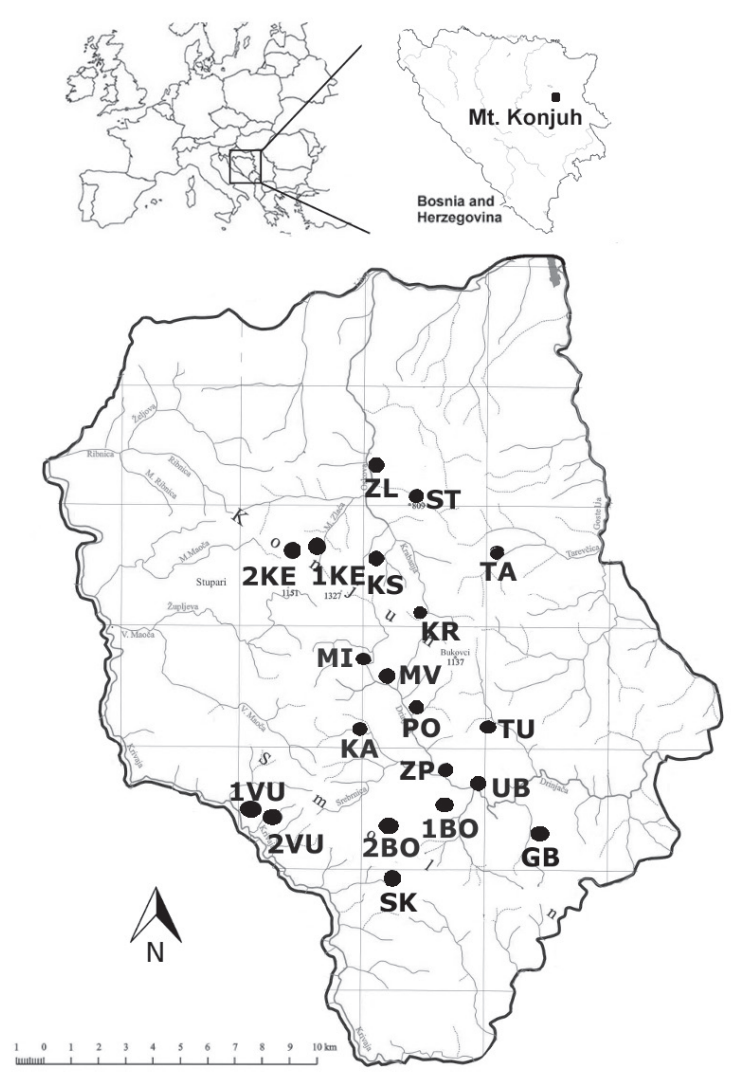

Fig. 1. Location of the study sites presented by spring codes on the Mt. Konjuh in Bosnia and Herzegovina.

transects under an Olympus BX41 light microscope (Olympus Corporation, Japan) at a magnification of 1000x. Quantitative data on diatoms and non-diatoms were integrated according to Stevenson and Bahls (1999). Because of their estimation per different units, and the need for uniformity for statistical analysis, the absolute cell numbers were transformed into relative (percentage) abundances. In addition to the abundance, which shows the average percentage of the taxa within samples, data on the taxa frequency are also given to provide information on the occurrence of taxa in the analyzed group of samples.

Identification was done following Starmach (1985), Cvijan and Blaženčić (1996), Krammer and Lange-Bertalot (1986, 1988, 2000, 2004 a,b), Komárek and Anagnostidis $(1998,2005)$, Krammer (2000, 2002), John et al. (2002), Hofmann et al. (2011) and Lange-Bertalot et al. (2017).

\section{Data processing and statistical analyses}

Diversity was calculated by using species richness $(S)$ as the number of identified taxa, the Shannon-Wiener index of species diversity, $\mathrm{H}^{\prime}(\mathrm{ln})$, (Shannon and Weaver 1949) and Pielou's index of equitability, J' (Krebs 1999). Ecological requirements of diatoms for moisture, $\mathrm{pH}$ and trophic status were obtained from van Dam et al. (1994). The diatom trophic index (TI) by Rott et al. (1999), the Croatian trophic diatom index $\left(\mathrm{TID}_{\mathrm{HR}}\right)$ and the Ecological Quality Ratio - EQR
(Anonymous 2013, 2016) were used in the evaluation of trophic and ecological status of spring ecosystems.

All data were transformed by using the square root function prior to statistical analyses. Non-metric multidimensional scaling (NMDS) and hierarchical group average clustering based on mean values of the relative algal abundance for each spring were used for spring ordering. Similarity profile analysis (SIMPROF) with iterative permutation procedure (999 permutations) was used for the detection of boundaries among clusters. The ordination was conducted on the Bray-Curtis similarity matrix of species data (Legendre and Legendre 1998). Normal distribution of the main physical and chemical parameters and values of diversity indices were tested by the Kolmogorov-Smirnov test in order to select parametric or nonparametric test. The significance of differences in the main physical and chemical factors between springs emerging from two different lithologies (ophiolites vs. limestone) was analyzed using the nonparametric Mann-Whitney U test $(\mathrm{p}<0.05)$. Parametric $\mathrm{t}$-test was used to test for differences between diversity indices in springs from contrasting lithologies. The IndVal analysis (Dufrene and Legendre 1997) was used to identify characteristic species of: i) springs on different lithological substrata, and ii) different clusters. The significance of indicator value of each species was estimated by Monte Carlo permutation tests. Statistical analyses were performed in IBM SPSS Statistics for Windows version 22.0 (IBM Corp., U.S.A.), Primer 6.0 (Clarke and Warwick 2001), and PC-ORD 5 software packages (Grandin 2006).

\section{Results}

\section{Environmental variables}

Ecomorphological characteristics of springs, including spring names with short spring codes, location, shading, velocity and discharge are described in On-line Suppl. Tab. 1. Springs on ophiolites were characterized by low but relatively steady discharge $\left(\leq 2 \mathrm{~L} \mathrm{~s}^{-1}\right)$, water temperature below $8{ }^{\circ} \mathrm{C}$, low turbidity, low calcium content, high magnesium, and high dissolved oxygen concentrations (On-line Suppl. Tab. 2). On the other hand, springs on carbonates (limestone) had an unstable discharge varying from complete drying up to $150 \mathrm{~L} \mathrm{~s}^{-1}$ (On-line Suppl. Tab. 1). Carbonate rheocrenic springs had higher values of oxygen concentration than the rheohelocrenes, neutral to slightly alkaline water, and high calcium content. High nitrate concentrations (up to $18 \mathrm{mg} \mathrm{L}^{-1}$ ) were noted in several springs independently from the lithology. The values of alkalinity and conductivity ranged from 16 to $70 \mathrm{mg} \mathrm{CaCO}_{3} \mathrm{~L}^{-1}$, and $136-584 \mu \mathrm{S} \mathrm{cm}^{-1}$, respectively (On-line Suppl. Tab. 2). Springs emerging from ophiolites showed higher values of temperature, $\mathrm{pH}$, magnesium and lower values of conductivity, discharge, sulphates and calcium than springs on carbonates (On-line Suppl. Tab. 3). During field studies many negative anthropogenic influences were noted. The most pronounced effects are alterations of morphology of the spring area for the purpose of water abstraction, deforestation with accompanying effects 
of tree transport, road infrastructure building and trampling by cattle.

\section{Species composition, diversity, ecological preferences of diatoms and trophic status}

A total of 234 algal taxa were identified in 196 samples collected from 20 springs. All identified taxa are listed in Tab. 1. The most abundant algal groups were diatoms and cyanobacteria, with 187 and 34 taxa, respectively. The other algal classes were Xanthophyceae (3), Florideophyceae, Ulvophyceae, Chlorophyceae and Conjugatophyceae (2 in each), Chrysophyceae and Klebsormidiophyceae (1 in each). Total number of recorded taxa ranged between 46 (rheocrenic spring on ophiolite - 2KE) and 76 (rheohelocrenic spring on ophiolite - SK). The most common algal genera were Gomphonema (17), Nitzschia (15), Navicula (11), Phormidium (9), Cymbella (9), and Achnanthidium (8). The species Achnanthidium minutissimum, Planothidium lanceolatum, Meridion circulare, Cocconeis pseudolineata and Amphora pediculus were recorded in more than a half of all investigated springs. Besides the above mentioned, also very abundant were Cocconeis lineata, Gomphonema micropus, Cocconeis euglypta, Odontidium mesodon and Tapinothrix varians.

Hierarchical group average clustering and the SIMPROF test identified 6 assemblages (Fig. 2) mainly related to spring type (rheocrenes and rheohelocrenes) and geological substratum (ophiolites, carbonates, and ophiolitic melange). Species contribution to the clusters was described by the SIMPER analysis (On-line Suppl. Tab. 4), and statistically significant indicator species $(\mathrm{p}<0.05)$ for each cluster provided by the IndVal analysis are listed in Fig. 2. Species indicator values and contribution of the most abundant species could not be technically estimated for springs MV (cluster 1) and ZL (cluster 6), because the first and the sixth cluster included only one spring. However, the most frequent and abundant species in spring MV (cluster 1) were Achnanthidium minutissimum, Achnanthidium pyrenaicum, Odontidium mesodon, Nitzschia fonticola and Amphora pediculus, whilst the most abundant and frequent species in spring ZL (cluster 6) were Meridion circulare, Gomphonema micropus, Eunotia soleirolii, Nitzschia dubia and Ammatoidea sp. Cluster 2 (rheocrenes on ophiolites) was characterized by the xerotolerant diatom Humidophilla perpusilla, distinguished by both IndVal and SIMPER analysis. Taxa distinguished by IndVal were the eutraphentic diatom Gomphonema parvulum and the cyanoprokaryote Pseudanabaena sp. SIMPER analysis singled out several species with high contributions: Planothidium lanceolatum, Chlorogloea microcystoides, Meridion circulare, Phormidium formosum, and other taxa listed in On-line Suppl. Tab. 4. Rheocrenes on carbonates (cluster 3) were characterized by Rhoicosphenia abbreviata and Nitzschia fonticola (given by IndVal), and a high contribution of the rheophilic cyanobacterium Tapinothrix varians, the crenophilous diatoms $M$. circulare and Odontidium mesodon, and the epiphytic diatom species P. lanceolatum and Cocconeis lineata. Cluster 4 comprised rheohelocrenes on ophiolites and carbonates, and was characterised by the diatoms Cocconeis pseudolineata, Encyonopsis cesatii, Diploneis fontanella, and Tryblionella angustata. IndVal also singled out Achnanthidium exile, Amphora lange-bertalotii var. tenuis, Cymbella hantzschiana, Gomphonema subclavatum, and Nitzschia capitellata. Representatives of shaded rheocrenes (cluster 5) were eutraphentic diatoms, such as Amphora pediculus, Encyonema minutum, Fallacia subhamulata, Gomphonema angustatum, Achnanthidium affine, and Cymbella diminuta.

According to NMDS, springs were separated by lithology, with carbonate springs distributed on one side and ophio-

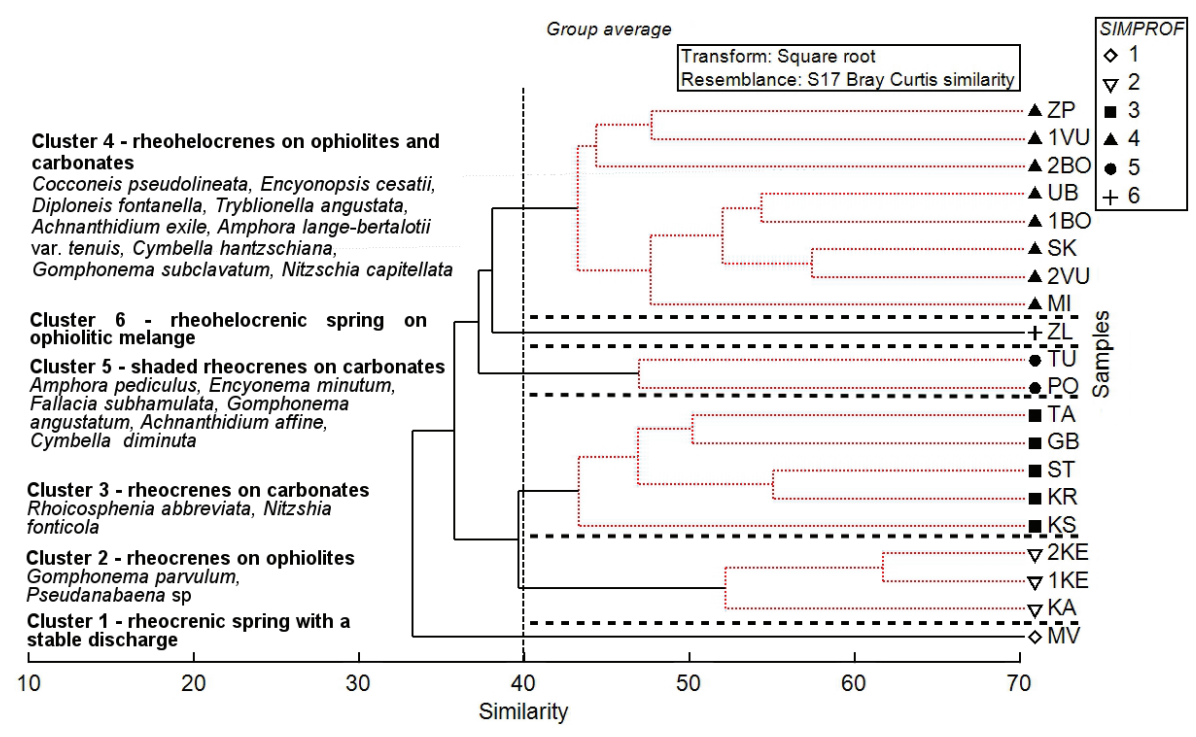

Fig. 2. Hierarchical group average clustering based on algal assemblages in the studied springs (presented by spring codes) on the Mt. Konjuh and characteristic species for each cluster identified by the IndVal analysis. Number of clusters was calculated with the SIMPROF analysis. 
Tab. 1. Algae of springs in the Mt. Konjuh, GS - geological substrata (O - ophiolites, C - carbonates, OM - ophiolitic melange); A - mean abundance per sample, \%; F - frequency per sample, \%; max. - maximum relative abundance per sample, \%; $\mathrm{N}$ - number of springs where the taxon was found.

\begin{tabular}{|c|c|c|c|c|c|}
\hline Taxa & GS & A & $\mathrm{F}$ & $\max$. & $\mathrm{N}$ \\
\hline \multicolumn{6}{|l|}{ Cyanophyceae } \\
\hline Ammatoidea sp. & $\mathrm{O}-\mathrm{C}-\mathrm{OM}$ & 8.5 & 7.1 & 50.0 & 4 \\
\hline Aphanocapsa minuta (Kylin) Whitton & $\mathrm{O}-\mathrm{C}$ & 0.4 & 1.0 & 0.7 & 2 \\
\hline Chamaesiphon confervicola A.Braun & $\mathrm{C}$ & 32.5 & 1.0 & 58.5 & 1 \\
\hline Chamaesiphon fuscus (Rostafinski) Hansgirg & $\mathrm{O}-\mathrm{C}$ & 1.9 & 2.0 & 5.9 & 3 \\
\hline Chamaesiphon incrustans Grunow & $\mathrm{C}$ & 1.1 & 1.5 & 3.1 & 1 \\
\hline Chamaesiphon polonicus (Rostafinski) Hansgirg & $\mathrm{O}$ & 9.1 & 3.6 & 28.6 & 5 \\
\hline Chamaesiphon sp. & $\mathrm{C}$ & 3.5 & 2.0 & 5.9 & 3 \\
\hline Chamaesiphon subglobosus (Rostafinski) Lemmermann & $\mathrm{O}$ & 0.2 & 0.5 & 0.2 & 1 \\
\hline Chlorogloea microcystoides Geitler & $\mathrm{O}-\mathrm{C}$ & 23.0 & 9.2 & 78.6 & 6 \\
\hline Chroococcaceae & $\mathrm{O}-\mathrm{C}-\mathrm{OM}$ & 21.2 & 4.1 & 72.7 & 5 \\
\hline Chroococcus sp. & $\mathrm{O}$ & 0.1 & 0.5 & 0.1 & 1 \\
\hline Chroococcus turgidus (Kützing) Nägeli & $\mathrm{O}-\mathrm{C}$ & 2.2 & 4.6 & 5.0 & 5 \\
\hline Clastidium setigerum O.Kirchner & $\mathrm{O}$ & 0.3 & 0.5 & 0.3 & 1 \\
\hline Cyanothece aeruginosa (Nägeli) Komárek & $\mathrm{O}$ & 0.03 & 0.5 & 0.0 & 1 \\
\hline Hassallia pulvinata Frémy & $\mathrm{O}-\mathrm{C}$ & 8.0 & 2.0 & 20.1 & 2 \\
\hline Hyella sp. & $\mathrm{C}$ & 0.3 & 0.5 & 0.3 & 1 \\
\hline Leptolyngbya perforans (Geitler) Anagnostidis et Komárek & $\mathrm{C}$ & 28.8 & 1.0 & 56.2 & 1 \\
\hline Leptolyngbya sp. & $\mathrm{O}-\mathrm{C}-\mathrm{OM}$ & 7.3 & 10.2 & 39.6 & 9 \\
\hline Lyngbya martensiana Meneghini ex Gomont & $\mathrm{O}-\mathrm{C}$ & 1.9 & 3.1 & 6.1 & 3 \\
\hline Lyngbya natans Hansgirg & $\mathrm{O}$ & 0.9 & 0.5 & 0.9 & 1 \\
\hline Oscillatoria sp. & $\mathrm{C}$ & 18.0 & 0.5 & 18.0 & 1 \\
\hline Phormidium corium Gomont ex Gomont & $\mathrm{O}$ & 4.8 & 1.0 & 8.6 & 1 \\
\hline Phormidium formosum (Bory de Saint-Vincent ex Gomont) Anagnostidis et Komárek & $\mathrm{O}-\mathrm{C}$ & 22.2 & 16.8 & 99.0 & 9 \\
\hline Phormidium griseoviolaceum (Skuja) Anagnostidis & $\mathrm{C}$ & 9.9 & 0.5 & 9.9 & 1 \\
\hline Phormidium incrustatum Gomont ex Gomont & $\mathrm{C}$ & 5.0 & 1.0 & 8.7 & 1 \\
\hline Phormidium kuetzingianum (Kirchner ex Hansgirg) Anagnostidis et Komárek & $\mathrm{C}$ & 1.5 & 1.0 & 1.6 & 1 \\
\hline Phormidium retzii Kützing ex Gomont & $\mathrm{O}-\mathrm{C}$ & 22.6 & 9.7 & 89.3 & 7 \\
\hline Phormidium rotheanum Itzigsohn & $\mathrm{O}$ & 2.4 & 1.0 & 2.8 & 1 \\
\hline Phormidium subfuscum Kützing ex Gomont & $\mathrm{O}-\mathrm{C}$ & 32.2 & 2.0 & 81.3 & 3 \\
\hline Phormidium tinctorium Kützing ex Gomont & $\mathrm{C}$ & 31.0 & 3.1 & 66.7 & 3 \\
\hline Pleurocapsa aurantiaca Geitler & $\mathrm{O}$ & 0.3 & 0.5 & 0.3 & 1 \\
\hline Pleurocapsa minor Hansgirg & $\mathrm{O}-\mathrm{C}$ & 8.7 & 3.6 & 35.6 & 4 \\
\hline Pseudanabaena sp. & $\mathrm{O}-\mathrm{C}$ & 6.1 & 6.1 & 19.6 & 3 \\
\hline Tapinothrix varians (Geitler) Bohunická et J.R.Johansen & $\mathrm{O}-\mathrm{C}$ & 36.9 & 28.1 & 98.7 & 14 \\
\hline \multicolumn{6}{|l|}{ Florideophyceae } \\
\hline Audouinella sp. & $\mathrm{C}$ & 21.4 & 5.1 & 70.0 & 3 \\
\hline Batrachospermum sp. & $\mathrm{C}$ & 6.8 & 0.5 & 6.8 & 1 \\
\hline \multicolumn{6}{|l|}{ Chrysophyceae } \\
\hline Phaeodermatium rivulare Hansgirg & $\mathrm{C}$ & 10.8 & 3.6 & 58.4 & 3 \\
\hline \multicolumn{6}{|l|}{ Xanthophyceae } \\
\hline Ophiocytium majus Nägeli & $\mathrm{O}$ & 0.1 & 0.5 & 0.1 & 1 \\
\hline Tribonema sp. & $\mathrm{O}-\mathrm{C}$ & 4.2 & 7.1 & 17.8 & 5 \\
\hline Vaucheria sp. & $\mathrm{C}$ & 4.9 & 8.7 & 24.7 & 3 \\
\hline \multicolumn{6}{|l|}{ Bacillariophyceae and Fragillariophyceae } \\
\hline Achnanthidium affine (Grunow) Czarnecki & $\mathrm{O}-\mathrm{C}-\mathrm{OM}$ & 2.8 & 14.8 & 28.4 & 9 \\
\hline Achnanthidium caledonicum (Lange-Bertalot) Lange-Bertalot & $\mathrm{O}-\mathrm{C}$ & 8.8 & 4.6 & 36.5 & 5 \\
\hline Achnanthidium dolomiticum M. Cantonati et $\mathrm{H}$. Lange-Bertalot & $\mathrm{O}$ & 2.4 & 1.5 & 3.3 & 1 \\
\hline Achnanthidium exile (Kützing) Heiberg & $\mathrm{O}-\mathrm{C}$ & 4.0 & 9.2 & 10.8 & 8 \\
\hline Achnanthidium minutissimum (Kützing) Czarnecki & $\mathrm{O}-\mathrm{C}-\mathrm{OM}$ & 20.6 & 87.8 & 82.1 & 20 \\
\hline Achnanthidium pyrenaicum (Hustedt) H.Kobayasi & $\mathrm{O}-\mathrm{C}-\mathrm{OM}$ & 7.4 & 29.1 & 53.4 & 13 \\
\hline Achnanthidium rosenstockii (Lange-Bertalot) Lange-Bertalot & $\mathrm{C}$ & 0.8 & 0.5 & 0.8 & 1 \\
\hline Achnanthidium straubianum (Lange-Bertalot) Lange-Bertalot & $\mathrm{O}-\mathrm{C}$ & 1.5 & 4.6 & 4.2 & 4 \\
\hline Adlafia bryophila (J.B.Petersen) Gerd Moser, Lange-Bertalot et D.Metzeltin & $\mathrm{O}-\mathrm{C}$ & 0.6 & 3.1 & 1.4 & 5 \\
\hline Adlafia minuscula (Grunow) Lange-Bertalot & $\mathrm{O}-\mathrm{C}-\mathrm{OM}$ & 0.9 & 12.2 & 2.8 & 11 \\
\hline Amphipleura pellucida (Kützing) Kützing & $\mathrm{O}-\mathrm{C}$ & 1.3 & 11.7 & 4.4 & 7 \\
\hline Amphora copulata (Kützing) Schoeman et R.E.M.Archibald & $\mathrm{C}$ & 1.5 & 0.5 & 1.5 & 1 \\
\hline Amphora indistincta Levkov & $\mathrm{O}-\mathrm{C}-\mathrm{OM}$ & 2.2 & 21.9 & 16.6 & 12 \\
\hline Amphora lange-bertalotii var. tenuis Levkov et Metzeltin & $\mathrm{O}-\mathrm{C}$ & 1.4 & 10.2 & 7.3 & 7 \\
\hline Amphora ovalis (Kützing) Kützing & $\mathrm{O}-\mathrm{C}-\mathrm{M}$ & 3.0 & 14.8 & 15.9 & 8 \\
\hline Amphora pediculus (Kützing) Grunow ex A.Schmidt & $\mathrm{O}-\mathrm{C}$ & 3.6 & 65.8 & 28.9 & 19 \\
\hline
\end{tabular}


Tab. 1. Continued

\begin{tabular}{|c|c|c|c|c|c|}
\hline Taxa & GS & $\mathrm{A}$ & $\mathrm{F}$ & max. & $\mathrm{N}$ \\
\hline Brachysira vitrea (Grunow) R.Ross & $\mathrm{C}$ & 0.7 & 0.5 & 0.7 & 1 \\
\hline Brebissonia lanceolata (C.Agardh) Mahoney et Reimer & $\mathrm{O}-\mathrm{C}$ & 3.0 & 8.2 & 27.6 & 7 \\
\hline Caloneis alpestris (Grunow) Cleve & $\mathrm{C}$ & 0.1 & 0.5 & 0.1 & 1 \\
\hline Caloneis fontinalis (Grunow) Lange-Bertalot et Reichardt & $\mathrm{O}-\mathrm{C}-\mathrm{M}$ & 0.8 & 28.6 & 4.1 & 18 \\
\hline Caloneis silicula (Ehrenberg) Cleve & $\mathrm{O}-\mathrm{C}$ & 0.4 & 3.1 & 0.5 & 4 \\
\hline Caloneis sp. & C & 0.5 & 0.5 & 0.5 & 1 \\
\hline Caloneis tenuis (W.Gregory) Krammer & $\mathrm{O}-\mathrm{C}-\mathrm{M}$ & 0.5 & 4.6 & 0.9 & 7 \\
\hline Chamaepinnularia parsura (Hustedt) C.E.Wetzel et Ector & $\mathrm{C}$ & 0.2 & 0.5 & 0.2 & 1 \\
\hline Cocconeis disculus (Schumann) Cleve & $\mathrm{O}$ & 3.0 & 3.1 & 8.2 & 1 \\
\hline Cocconeis pediculus Ehrenberg & $\mathrm{C}$ & 0.2 & 1.0 & 0.2 & 1 \\
\hline Cocconeis euglypta Ehrenberg & $\mathrm{O}-\mathrm{C}-\mathrm{OM}$ & 5.2 & 39.8 & 38.0 & 15 \\
\hline Cocconeis placentula var. klinoraphis Geitler & $\mathrm{O}$ & 0.5 & 0.5 & 0.5 & 1 \\
\hline Cocconeis lineata Ehrenberg & $\mathrm{O}-\mathrm{C}-\mathrm{OM}$ & 6.9 & 58.7 & 85.5 & 18 \\
\hline Cocconeis pseudolineata (Geitler) Lange-Bertalot & $\mathrm{O}-\mathrm{C}-\mathrm{OM}$ & 6.7 & 57.7 & 74.7 & 20 \\
\hline Cymatopleura solea var. apiculata (W.Smith) Ralfs & $\mathrm{C}$ & 0.4 & 1.5 & 0.8 & 3 \\
\hline Cymbella affinis Kützing & $\mathrm{O}-\mathrm{C}$ & 1.7 & 8.7 & 12.5 & 5 \\
\hline Cymbella cymbiformis C.Agardh & $\mathrm{O}-\mathrm{C}$ & 7.4 & 4.6 & 29.2 & 2 \\
\hline Cymbella excisiformis Krammer & $\mathrm{O}$ & 0.8 & 2.0 & 1.4 & 1 \\
\hline Cymbella diminuta (Grunow) Reichardt & $\mathrm{C}$ & 0.9 & 3.1 & 2.1 & 2 \\
\hline Cymbella hantzschiana Krammer & $\mathrm{O}-\mathrm{C}$ & 1.7 & 13.8 & 4.7 & 10 \\
\hline Cymbella laevis Nägeli & $\mathrm{C}$ & 1.2 & 1.0 & 2.1 & 1 \\
\hline Cymbella parva (W.Smith) Kirchner & $\mathrm{O}-\mathrm{C}$ & 1.3 & 12.2 & 5.5 & 9 \\
\hline Cymbella tridentina Lange-Bertalot, M.Cantonati et A.Scalfi & $\mathrm{O}$ & 5.1 & 3.1 & 20.1 & 2 \\
\hline Cymbella vulgata Krammer & $\mathrm{C}$ & 0.2 & 1.0 & 0.3 & 2 \\
\hline Cymbopleura amphicephala (Nägeli) Krammer & $\mathrm{O}$ & 0.8 & 2.6 & 1.6 & 1 \\
\hline Cymbopleura florentina (Grunow) K.Krammer & $\mathrm{O}$ & 0.4 & 1.5 & 0.7 & 1 \\
\hline Cymbopleura subaequalis (Grunow) Krammer & $\mathrm{C}$ & 0.7 & 0.5 & 0.7 & 1 \\
\hline Cymbopleura subaustriaca Krammer & $\mathrm{O}-\mathrm{C}-\mathrm{OM}$ & 1.9 & 5.1 & 7.0 & 3 \\
\hline Decussata hexagona (Torka) Lange-Bertalot & $\mathrm{C}$ & 0.4 & 0.5 & 0.4 & 1 \\
\hline Delicata minuta K.Krammer & $\mathrm{O}-\mathrm{C}$ & 0.4 & 1.5 & 0.6 & 2 \\
\hline Denticula kuetzingii Grunow & $\mathrm{O}-\mathrm{C}$ & 0.9 & 4.1 & 2.2 & 5 \\
\hline Denticula tenuis Kützing & $\mathrm{O}-\mathrm{C}$ & 3.2 & 16.8 & 21.8 & 9 \\
\hline Humidophila contenta (Grunow) Lowe, Kociolek, Johansen, Van de Vijver, Lange-Bertalot et Kopalová & $\mathrm{O}-\mathrm{C}$ & 0.5 & 8.7 & 1.9 & 9 \\
\hline $\begin{array}{l}\text { Humidophila paracontenta var. magisconcava (Lange-Bertalot) Lowe, Kociolek, Johansen, Van de Vijver, } \\
\text { Lange-Bertalot et Kopalová }\end{array}$ & $\mathrm{O}-\mathrm{C}$ & 1.3 & 9.2 & 6.7 & 11 \\
\hline Humidophila perpusilla (Grunow) Lowe, Kociolek, Johansen,Van deVijver, Lange-Bertalot et Kopalová & $\mathrm{O}-\mathrm{C}$ & 5.7 & 27.0 & 76.7 & 14 \\
\hline Humidophila sp. & $\mathrm{OM}$ & 0.5 & 0.5 & 0.5 & 1 \\
\hline Odontidium hyemale (Roth) Kützing & $\mathrm{O}-\mathrm{C}$ & 1.1 & 3.6 & 2.2 & 5 \\
\hline Odontidium mesodon (Ehrenberg) Kützing & $\mathrm{O}-\mathrm{C}-\mathrm{OM}$ & 7.4 & 35.2 & 65.2 & 17 \\
\hline Diploneis elliptica (Kützing) Cleve & $\mathrm{O}-\mathrm{C}$ & 1.0 & 4.6 & 4.1 & 4 \\
\hline Diploneis fontanella Lange-Bertalot & $\mathrm{O}-\mathrm{C}-\mathrm{OM}$ & 1.0 & 19.9 & 3.4 & 14 \\
\hline Diploneis krammeri Lange-Bertalot et E.Reichardt & $\mathrm{O}-\mathrm{C}-\mathrm{OM}$ & 1.3 & 26.0 & 13.2 & 16 \\
\hline Diploneis minuta J.B.Petersen & $\mathrm{O}-\mathrm{C}$ & 0.5 & 3.1 & 0.7 & 3 \\
\hline Encyonema hebridicum (Gregory) Grunow & $\mathrm{O}-\mathrm{C}$ & 0.6 & 2.6 & 1.2 & 3 \\
\hline Encyonema minutum (Hilse) D.G.Mann & $\mathrm{O}-\mathrm{C}$ & 0.7 & 4.6 & 2.3 & 5 \\
\hline Encyonema neogracile Krammer & $\mathrm{O}$ & 0.4 & 0.5 & 0.4 & 1 \\
\hline Encyonema silesiacum (Bleisch) D.G.Mann & $\mathrm{O}-\mathrm{C}$ & 0.7 & 5.6 & 1.6 & 5 \\
\hline Encyonema triangulum (Ehrenberg) Kützing & $\mathrm{O}$ & 0.6 & 1.0 & 0.7 & 1 \\
\hline Encyonema ventricosum (C.Agardh) Grunow & $\mathrm{O}-\mathrm{C}-\mathrm{OM}$ & 2.5 & 16.3 & 30.5 & 9 \\
\hline Encyonopsis cesatii (Rabenhorst) Krammer & $\mathrm{O}-\mathrm{C}$ & 3.9 & 35.2 & 24.3 & 15 \\
\hline Encyonopsis fonticola (Hustedt) Krammer & $\mathrm{O}-\mathrm{C}$ & 2.9 & 10.2 & 10.2 & 9 \\
\hline Encyonopsis krammeri Reichardt & $\mathrm{O}-\mathrm{C}-\mathrm{OM}$ & 1.0 & 16.8 & 5.5 & 12 \\
\hline Encyonopsis microcephala (Grunow) Krammer & $\mathrm{O}$ & 1.1 & 3.1 & 2.5 & 1 \\
\hline Encyonopsis minuta Krammer et E.Reichardt & M & 0.7 & 3.1 & 1.8 & 1 \\
\hline Epithemia argus (Ehrenberg) Kützing & $\mathrm{O}-\mathrm{C}-\mathrm{OM}$ & 1.4 & 2.0 & 4.3 & 1 \\
\hline Epithemia frickei Krammer & $\mathrm{O}-\mathrm{C}$ & 1.0 & 1.5 & 2.2 & 3 \\
\hline Epithemia turgida (Ehrenberg) Kützing & $\mathrm{C}$ & 1.1 & 2.6 & 3.3 & 1 \\
\hline Eucocconeis flexella (Kützing) Meister & $\mathrm{O}$ & 0.6 & 1.5 & 0.9 & 2 \\
\hline Eucocconeis laevis (Østrup) Lange-Bertalot & $\mathrm{O}-\mathrm{C}-\mathrm{OM}$ & 0.7 & 6.1 & 2.7 & 8 \\
\hline Eunotia ambivalens Lange-Bertalot et Tagliaventi & $\mathrm{O}$ & 1.4 & 1.0 & 1.6 & 1 \\
\hline Eunotia arcubus Nörpel et Lange-Bertalot & $\mathrm{O}-\mathrm{C}$ & 7.8 & 11.2 & 60.7 & 7 \\
\hline Eunotia soleirolii (Kützing) Rabenhorst & $\mathrm{O}-\mathrm{OM}$ & 1.4 & 9.2 & 4.1 & 4 \\
\hline Fallacia lange-bertalotii (E.Reichardt) E.Reichardt & $\mathrm{C}$ & 0.7 & 2.0 & 1.2 & 2 \\
\hline
\end{tabular}


Tab. 1. Continued

\begin{tabular}{|c|c|c|c|c|c|}
\hline Taxa & GS & A & $\mathrm{F}$ & max. & $\mathrm{N}$ \\
\hline Fallacia subhamulata (Grunow) D.G.Mann & $\mathrm{C}$ & 1.4 & 4.1 & 7.8 & 4 \\
\hline Fragilaria amphicephaloides Lange-Bertalot & $\mathrm{O}$ & 1.4 & 2.0 & 1.9 & 1 \\
\hline Fragilaria recapitellata $\mathrm{H}$. Lange-Bertalot et $\mathrm{D}$. Metzeltin & $\mathrm{C}$ & 4.1 & 5.6 & 26.9 & 3 \\
\hline Frustulia vulgaris (Thwaites) De Toni & $\mathrm{C}-\mathrm{OM}$ & 0.3 & 3.1 & 0.5 & 4 \\
\hline Gomphonema angustatum (Kützing) Rabenhorst & $\mathrm{C}-\mathrm{OM}$ & 1.5 & 12.8 & 11.6 & 8 \\
\hline Gomphonema angustivalva E. Reichardt & $\mathrm{O}-\mathrm{C}$ & 3.3 & 1.0 & 6.2 & 2 \\
\hline Gomphonema angustum C.Agardh & $\mathrm{O}-\mathrm{C}-\mathrm{OM}$ & 1.7 & 27.6 & 10.5 & 14 \\
\hline Gomphonema brebissonii Kützing & $\mathrm{O}$ & 0.4 & 1.0 & 0.4 & 1 \\
\hline Gomphonema elegantissimum Reichardt et Lange-Bertalot & $\mathrm{O}-\mathrm{C}$ & 4.7 & 18.4 & 42.6 & 8 \\
\hline Gomphonema exilissimum (Grunow) Lange-Bertalot et E.Reichardt & $\mathrm{O}-\mathrm{C}$ & 1.4 & 15.8 & 7.1 & 11 \\
\hline Gomphonema micropus Kützing & $\mathrm{O}-\mathrm{C}-\mathrm{OM}$ & 2.0 & 44.9 & 23.3 & 17 \\
\hline Gomphonema occultum E.Reichardt et Lange-Bertalot & $\mathrm{C}$ & 0.6 & 2.0 & 1.2 & 2 \\
\hline Gomphonema olivaceum (Hornemann) Brébisson & $\mathrm{C}$ & 0.6 & 4.6 & 1.8 & 2 \\
\hline Gomphonema olivaceoides Hustedt & $\mathrm{C}$ & 0.8 & 0.5 & 0.8 & 1 \\
\hline Gomphonema parvulum (Kützing) Kützing & $\mathrm{O}-\mathrm{C}-\mathrm{OM}$ & 1.8 & 18.4 & 11.7 & 15 \\
\hline Gomphonema procerum Reichardt et Lange-Bertalot & $\mathrm{O}-\mathrm{C}$ & 0.2 & 1.5 & 0.5 & 2 \\
\hline Gomphonema pseudoaugur Lange-Bertalot & $\mathrm{O}$ & 0.3 & 0.5 & 0.3 & 1 \\
\hline Gomphonema pseudobohemicum Lange-Bertalot et E.Reichardt & $\mathrm{C}$ & 1.6 & 2.6 & 3.7 & 2 \\
\hline Gomphonema pumilum (Geitler) Bohunická et J.R.Johansen & $\mathrm{OM}$ & 3.1 & 24.5 & 46.0 & 12 \\
\hline Gomphonema subclavatum (Grunow) Grunow & $\mathrm{O}-\mathrm{C}-\mathrm{OM}$ & 0.6 & 12.2 & 1.3 & 10 \\
\hline Gomphonema tergestinum (Grunow) Fricke & $\mathrm{O}-\mathrm{C}$ & 2.0 & 12.8 & 7.0 & 10 \\
\hline Grunowia sinuata (Thwaites) Rabenhorst & $\mathrm{O}-\mathrm{C}$ & 1.6 & 3.1 & 5.9 & 2 \\
\hline Gyrosigma acuminatum (Kützing) Rabenhorst & $\mathrm{C}$ & 6.1 & 4.1 & 14.5 & 1 \\
\hline Gyrosigma obtusatum (Sullivant \& Wormley) C.S.Boyer & $\mathrm{C}$ & 1.5 & 2.0 & 2.4 & 1 \\
\hline Halamphora normanii (Rabenhorst) Levkov & $\mathrm{O}-\mathrm{C}$ & 1.2 & 3.1 & 3.6 & 4 \\
\hline Halamphora veneta (Kützing) Levkov & $\mathrm{O}$ & 0.7 & 0.5 & 0.7 & 1 \\
\hline Hannaea arcus (Ehrenberg) R.M.Patrick & $\mathrm{C}$ & 0.1 & 0.5 & 0.1 & 1 \\
\hline Hantzschia amphioxys (Ehrenberg) Grunow & $\mathrm{O}-\mathrm{C}$ & 0.5 & 4.1 & 0.9 & 3 \\
\hline Luticola mutica (Kützing) D.G.Mann & C-OM & 0.8 & 3.1 & 2.0 & 4 \\
\hline Meridion circulare (Greville) C.Agardh & $\mathrm{O}-\mathrm{C}-\mathrm{OM}$ & 5.9 & 64.3 & 38.7 & 20 \\
\hline Navicula antonii Lange-Bertalot & $\mathrm{C}$ & 1.7 & 21.9 & 7.5 & 10 \\
\hline Navicula cariocincta Lange-Bertalot & $\mathrm{C}$ & 1.0 & 3.6 & 3.1 & 3 \\
\hline Navicula cataracta-rheni Lange-Bertalot & $\mathrm{O}$ & 2.0 & 6.1 & 8.9 & 3 \\
\hline Navicula cryptotenella Lange-Bertalot & $\mathrm{O}-\mathrm{C}-\mathrm{OM}$ & 1.7 & 12.8 & 22.4 & 8 \\
\hline Navicula cryptotenelloides Lange-Bertalot & $\mathrm{O}$ & 0.8 & 0.5 & 0.8 & 1 \\
\hline Navicula gregaria Donkin & $\mathrm{C}$ & 1.1 & 0.5 & 1.1 & 1 \\
\hline Navicula leistikowii Lange-Bertalot & $\mathrm{O}-\mathrm{C}$ & 0.9 & 13.8 & 2.7 & 9 \\
\hline Navicula oblonga (Kützing) Kützing & $\mathrm{O}$ & 0.6 & 0.5 & 0.6 & 1 \\
\hline Navicula radiosa Kützing & $\mathrm{O}-\mathrm{C}$ & 1.2 & 17.9 & 4.0 & 11 \\
\hline Navicula sp. & $\mathrm{C}$ & 1.9 & 4.1 & 10.3 & 3 \\
\hline Navicula tripunctata (O.F.Müller) Bory de Saint-Vincent & $\mathrm{O}-\mathrm{C}$ & 1.2 & 17.3 & 6.2 & 9 \\
\hline Naviculadicta geisslerae (Jahn) Jahn & $\mathrm{O}$ & 0.7 & 0.5 & 0.7 & 1 \\
\hline Naviculadicta tridentula (Krasske) Lange-Bertalot & $\mathrm{O}$ & 0.6 & 2.6 & 1.6 & 3 \\
\hline Naviculadicta sp. & $\mathrm{C}$ & 4.4 & 0.5 & 4.4 & 1 \\
\hline Neidiomorpha binodiformis (K.Krammer) M.Cantonati, H.Lange-Bertalot et N.Angeli & $\mathrm{O}-\mathrm{C}$ & 0.3 & 1.5 & 0.4 & 2 \\
\hline Neidium longiceps (Gregory) Ross & $\mathrm{O}-\mathrm{C}$ & 0.3 & 2.0 & 0.5 & 3 \\
\hline Nitzschia amphibia Grunow & $\mathrm{O}-\mathrm{C}$ & 1.6 & 7.1 & 7.2 & 8 \\
\hline Nitzschia capitellata Hustedt & $\mathrm{O}-\mathrm{C}$ & 0.8 & 5.6 & 1.8 & 7 \\
\hline Nitzschia dissipata (Kützing) Grunow & $\mathrm{O}-\mathrm{C}$ & 1.0 & 15.3 & 4.1 & 11 \\
\hline Nitzschia dubia W.Smith & $\mathrm{O}-\mathrm{C}-\mathrm{OM}$ & 2.1 & 14.3 & 11.2 & 7 \\
\hline Nitzschia fonticola (Grunow) Grunow & $\mathrm{C}$ & 4.5 & 10.2 & 33.5 & 6 \\
\hline Nitzschia frustulum (Kützing) Grunow & $\mathrm{O}-\mathrm{C}-\mathrm{OM}$ & 1.2 & 10.2 & 3.4 & 9 \\
\hline Nitzschia heufleriana Grunow & $\mathrm{C}$ & 0.6 & 1.5 & 0.8 & 1 \\
\hline Nitzschia inconspicua Grunow & $\mathrm{O}-\mathrm{C}$ & 0.7 & 3.6 & 1.3 & 6 \\
\hline Nitzschia linearis (C.Agardh) W.Smith & $\mathrm{O}-\mathrm{C}$ & 2.7 & 31.6 & 58.5 & 16 \\
\hline Nitzschia palea (Kützing) W.Smith & $\mathrm{O}-\mathrm{C}$ & 2.4 & 14.8 & 12.0 & 12 \\
\hline Nitzschia palea var. tenuirostris Grunow & $\mathrm{C}-\mathrm{OM}$ & 4.8 & 7.1 & 31.6 & 5 \\
\hline Nitzschia oligotraphenta (Lange-Bertalot) Lange-Bertalot & $\mathrm{C}$ & 0.5 & 0.5 & 0.5 & 1 \\
\hline Nitzschia sp.1 & $\mathrm{C}$ & 2.6 & 0.5 & 2.6 & 1 \\
\hline Nitzschia sp.2 & $\mathrm{O}$ & 0.9 & 0.5 & 0.9 & 1 \\
\hline Nitzschia umbonata (Ehrenberg) Lange-Bertalot & $\mathrm{C}$ & 1.1 & 0.5 & 1.1 & 1 \\
\hline
\end{tabular}


Tab. 1. Continued

\begin{tabular}{|c|c|c|c|c|c|}
\hline Taxa & GS & A & F & max. & $\mathrm{N}$ \\
\hline Nupela lapidosa (Krasske) Lange-Bertalot & $\mathrm{O}$ & 0.7 & 0.5 & 0.7 & 1 \\
\hline Orthoseira roeseana (Rabenhorst) O'Meara & $\mathrm{O}-\mathrm{C}$ & 0.7 & 2.0 & 1.4 & 3 \\
\hline Pinnularia acutobrebissonii Kulikovskiy, Lange-Bertalot et Metzeltin & $\mathrm{O}$ & 0.9 & 2.0 & 1.1 & 1 \\
\hline Pinnularia borealis Ehrenberg & $\mathrm{O}$ & 0.5 & 2.6 & 0.7 & 2 \\
\hline Pinnularia gibba Ehrenberg & $\mathrm{C}$ & 0.4 & 0.5 & 0.4 & 1 \\
\hline Pinnularia kuetzingii K.Krammer & $\mathrm{O}$ & 0.7 & 2.0 & 0.8 & 1 \\
\hline Pinnularia sp. & $\mathrm{OM}$ & 0.5 & 0.5 & 0.5 & 1 \\
\hline Pinnularia subcapitata var. elongata Krammer & $\mathrm{O}$ & 1.0 & 2.0 & 2.0 & 1 \\
\hline Pinnularia subrupestris K.Krammer & $\mathrm{O}-\mathrm{C}-\mathrm{OM}$ & 1.1 & 6.1 & 3.3 & 7 \\
\hline Placoneis paraelginensis Lange-Bertalot & $\mathrm{O}-\mathrm{M}$ & 0.3 & 3.1 & 0.5 & 5 \\
\hline Platessa conspicua (A. Mayer) Lange-Bertalot & M & 2.8 & 1.5 & 8.1 & 1 \\
\hline Platessa lutheri (Hustedt) Potapova & $\mathrm{C}$ & 0.9 & 1.5 & 1.6 & 1 \\
\hline Planothidium dubium (Grunow) Round et Bukhtiyarova & M & 1.4 & 22.4 & 10.3 & 8 \\
\hline Planothidium frequentissimum (Lange-Bertalot) Round et L.Bukhtiyarova & $\mathrm{O}-\mathrm{C}-\mathrm{OM}$ & 1.8 & 21.9 & 6.6 & 18 \\
\hline Planothidium joursacense (Héribaud-Joseph) Lange-Bertalot & $\mathrm{O}$ & 3.6 & 0.5 & 3.6 & 1 \\
\hline Planothidium lanceolatum (Brébisson ex Kützing) Lange-Bertalot & $\mathrm{O}-\mathrm{C}-\mathrm{OM}$ & 10.7 & 86.7 & 64.9 & 20 \\
\hline Planothidium minutissimum (Krasske) E.A.Morales & $\mathrm{C}-\mathrm{OM}$ & 10.1 & 1.0 & 15.8 & 2 \\
\hline Planothidium reichardtii Lange-Bertalot et Werum & $\mathrm{O}-\mathrm{C}$ & 1.0 & 5.1 & 3.9 & 8 \\
\hline Platessa holsatica (Hust.) Lange-Bertalot & $\mathrm{C}-\mathrm{OM}$ & 4.4 & 4.1 & 28.9 & 5 \\
\hline Psammothidium grischunum (Wuthrich) L.Bukhtiyarova et Round & $\mathrm{C}$ & 3.7 & 13.3 & 15.7 & 6 \\
\hline Psammothidium subatomoides (Hustedt) Bukhtiyarova et Round & $\mathrm{O}-\mathrm{C}-\mathrm{OM}$ & 1.5 & 8.7 & 10.7 & 7 \\
\hline Pseudostaurosira brevistriata (Grunow) Williams et Round & $\mathrm{C}$ & 0.1 & 0.5 & 0.1 & 1 \\
\hline Reimeria sinuata (Gregory) Kociolek et Stoermer & $\mathrm{C}$ & 0.3 & 1.0 & 0.4 & 1 \\
\hline Rhoicosphenia abbreviata (C.Agardh) Lange-Bertalot & $\mathrm{C}$ & 0.7 & 9.2 & 2.9 & 5 \\
\hline Rhopalodia parallela (Grunow) O. Müller & $\mathrm{O}-\mathrm{C}$ & 0.5 & 5.1 & 0.8 & 3 \\
\hline Rossithidium anastasiae (Kaczmarska) Potapova & $\mathrm{O}-\mathrm{C}-\mathrm{OM}$ & 2.8 & 11.2 & 15.3 & 7 \\
\hline Rossithidium petersenii (Hustedt) Round et Bukhtiyarova & $\mathrm{O}-\mathrm{C}$ & 1.4 & 6.6 & 3.0 & 6 \\
\hline Rossithidium pusillum (Grunow) Round et L.Bukhtiyarova & $\mathrm{C}$ & 0.2 & 1.0 & 0.3 & 1 \\
\hline Sellaphora seminulum (Grunow) D.G. Mann & $\mathrm{O}-\mathrm{C}$ & 0.6 & 4.6 & 1.7 & 6 \\
\hline Sellaphora pseudopupula (Krasske) Lange-Bertalot & $\mathrm{C}$ & 0.4 & 1.5 & 0.7 & 3 \\
\hline Sellaphora pupula (Kützing) Mereschkovsky & $\mathrm{C}$ & 0.7 & 9.2 & 2.4 & 6 \\
\hline Sellaphora stroemii (Hustedt) H.Kobayasi & $\mathrm{O}$ & 0.6 & 0.5 & 0.6 & 1 \\
\hline Sellaphora nigri (De Notaris) C.E. Wetzel et L. Ector & $\mathrm{O}-\mathrm{C}-\mathrm{OM}$ & 2.9 & 25.0 & 22.5 & 17 \\
\hline Simonsenia delognei (Grunow) Lange-Bertalot & $\mathrm{C}$ & 1.1 & 1.5 & 3.1 & 1 \\
\hline Staurosirella martyi (Héribaud) Morales et Manoylov & $\mathrm{C}$ & 0.1 & 0.5 & 0.1 & 1 \\
\hline Stauroneis anceps Ehrenberg & $\mathrm{O}-\mathrm{OM}$ & 0.3 & 2.6 & 0.4 & 3 \\
\hline Stauroneis jarensis Lange-Bertalot & $\mathrm{O}$ & 0.3 & 0.5 & 0.3 & 1 \\
\hline Stauroneis phoenicenteron (Nitzsch) Ehrenberg & $\mathrm{C}$ & 0.3 & 0.5 & 0.3 & 1 \\
\hline Stauroneis smithii Grunow & $\mathrm{O}-\mathrm{C}$ & 0.8 & 6.1 & 3.6 & 5 \\
\hline Surirella angusta Kützing & $\mathrm{O}-\mathrm{C}-\mathrm{OM}$ & 1.1 & 10.2 & 5.0 & 9 \\
\hline Surirella brebissonii var. kuetzingii Krammer et Lange-Bertalot & $\mathrm{O}$ & 0.2 & 0.5 & 0.2 & 1 \\
\hline Surirella linearis W.Smith & $\mathrm{O}-\mathrm{C}$ & 2.1 & 18.4 & 12.8 & 12 \\
\hline Surirella minuta Brébisson & $\mathrm{O}-\mathrm{C}$ & 1.0 & 4.6 & 2.9 & 5 \\
\hline Surirella spiralis Kützing & $\mathrm{O}$ & 0.7 & 0.5 & 0.7 & 1 \\
\hline Surirella terricola Lange-Bertalot et E.Alles & $\mathrm{O}-\mathrm{C}-\mathrm{OM}$ & 0.9 & 8.7 & 3.3 & 9 \\
\hline Tryblionella angustata W.Smith & $\mathrm{O}-\mathrm{C}$ & 3.0 & 10.7 & 13.9 & 11 \\
\hline Tryblionella angustatula (Lange-Bertalot) Cantonati et Lange-Bertalot & $\mathrm{O}$ & 1.2 & 3.6 & 3.1 & 3 \\
\hline Ulnaria ulna (Nitzsch) P.Compère & $\mathrm{O}-\mathrm{C}-\mathrm{OM}$ & 0.8 & 24.5 & 4.1 & 16 \\
\hline \multicolumn{6}{|l|}{ Chlorophyceae } \\
\hline Chaetophora sp. & $\mathrm{O}$ & 12.9 & 1.0 & 20.0 & 1 \\
\hline Microspora sp. & $\mathrm{O}$ & 0.6 & 0.5 & 0.6 & 1 \\
\hline \multicolumn{6}{|l|}{ Klebsormidiophyceae } \\
\hline Klebsormidium flaccidum (Kützing) P.C. Silva, K.R. Mattox et W.H. Blackwell & $\mathrm{O}$ & 3.2 & 1.5 & 5.1 & 1 \\
\hline \multicolumn{6}{|l|}{ Ulvophyceae } \\
\hline Ulothrix sp. & $\mathrm{O}$ & 0.3 & 0.5 & 0.3 & 1 \\
\hline Ulotrichales & $\mathrm{C}$ & 0.8 & 1.0 & 1.7 & 1 \\
\hline \multicolumn{6}{|l|}{ Conjugatophyceae } \\
\hline Cosmarium obtusatum (Schmidle) Schmidle & $\mathrm{O}$ & 0.1 & 1.5 & 0.1 & 1 \\
\hline Spirogyra sp. & $\mathrm{C}$ & 0.5 & 1.5 & 1.0 & 1 \\
\hline
\end{tabular}


lite springs on the other side of the plot (Fig. 3). The highest Shannon-Wiener diversity and the highest number of taxa were recorded from the epibryon sample of the spring SK on ophiolitic substratum (2.95 and 47 , respectively). The lowest diversity (0.08) and the lowest number of taxa (5) were found in the epilithon summer sample in the spring TU on carbonate substratum. The rheocrenic spring ST, characterised by the species Tapinothrix varians and Cocconeis linea$\mathrm{ta}$, had the lowest values of Pielou's Index, indicating several dominant and subdominant species in the sample and huge unevenness in species abundances. Parametric $t$-test performed on diversity indices of springs on different geological substrata did not show statistical differences ( $p>0.05)$. However, statistical differences for used trophic indices between two groups of springs were found (Rott's Trophic Index $-\mathrm{TI}, \mathrm{t}=-2.4, \mathrm{p}=0.02$, Croatian Diatom Trophic Index $-\mathrm{TID}_{\mathrm{HR}}, \mathrm{t}=2.75, \mathrm{p}=0.007$, and the Ecological quality ratio - EQR which is based on $\left.\mathrm{TID}_{\mathrm{HR}}\right)$. Higher mean values of trophic indices were noted in samples from ophiolites (Tab. 2). Spring ZL on ophiolitic melange was not considered for statistical analysis as it was the sole site on this geological substratum. Values of TI varied between 1.22 (spring MV) and 2.59 (spring ZL). Meso-eutrophic and eutrophic conditions were detected for eleven and eight springs, respectively. According to the TI, an oligotrophic status was assessed only for the spring MV. Assessment of ecological status of springs based on values of EQR and $\mathrm{TID}_{\mathrm{HR}}$ indicated good status for all localities, except for the spring MV (excellent status) and spring PO (relatively good status) (Tab. 2).

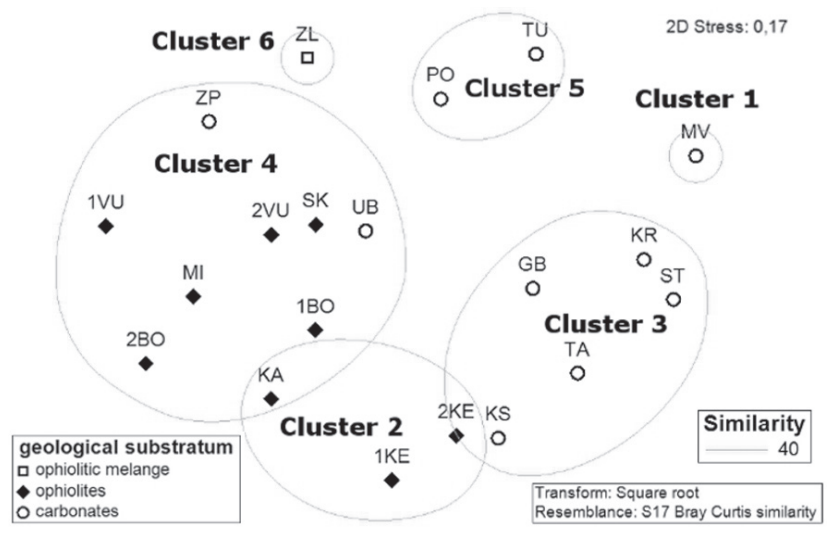

Fig. 3. Non-metric multidimensional scaling (NMDS) of sites (presented by spring codes) based on Bray-Curtis matrix of similarities of algal assemblages in relation to geological substratum and clusters.

Trophic preferences were found for 101 species (54\%), and were as follows: oligotraphentic (13.95\%, Encyonopsis cesatii, Gomphonema angustum, Surirella spiralis), oligo-mesotraphentic (13.9\%), mesotraphentic (9.9\%), meso-eutraphentic (12.9\%), eutraphentic (32.75\%, Amphora copulata, A. pediculus, Cocconeis spp., Navicula tripunctata), hypereutraphentic (3\%, Nitzschia palea, Nitzschia umbonata) and oligo - to eutraphentic (13.9\%).
Values of $\mathrm{pH}$ preferences were found for $58.3 \%$ of identified diatoms: $51.4 \%$ alkaliphilous (most frequent taxa: $A m$ phora pediculus, Cocconeis lineata, C. euglypta, Gomphonema angustum, G. micropus, Meridion circulare, Navicula tripunctata, Planothidium lanceolatum), 33.9\% circumneutral (e.g., A. minutissimum, Odontidium mesodon, Encyonema minutum, Encyonopsis cesatii, Navicula radiosa, etc.), 9.2\% acidophilous (e.g. Neidium affine var. longiceps, Pinnularia subcapitata var. elongata, Tryblionella angustata), and 5.5\% alkalibiontic (rarely occurring species such as Achnanthidium exile and Halamphora veneta).

Moisture-condition preference indicator values were found for $50.2 \%$ of the diatom taxa: $17 \%$ of taxa were classified as 'never, or only very rarely occurring outside water bodies' (Meridion circulare, Nitzschia fonticola, Ulnaria ulna), 21.3\% occurred 'mainly in water bodies, sometimes on wet places.' The highest number of species $(46.8 \%)$ ' occurred mainly on wet and moist places', while a low number of species $(11.7 \%)$ 'occurred mainly on wet and moist or temporarily dry places' (Caloneis tenuis, Diploneis fontanella, Luticola mutica). The lowest number of taxa (3.2\%) belongs to the category 'occurring nearly outside of water bodies' (Adlafia bryophila, Diploneis minuta, Halamphora veneta).

\section{Geological preferences}

Geological preferences of algae for the two main geological substrata, carbonates and ophiolites were analyzed. As only one site on this geological substratum was available, the spring on ophiolitic melange could not be included in statistical analyses. A total of 49 species (31\%) were found exclusively in samples taken from springs on ophiolites, and were mainly identified from one site only, and the most common (relative frequency $>3 \%$ ) were: Navicula cataracta-rheni, Chamaesiphon polonicus, Tryblionella angustatula, Cymbella tridentina, Cocconeis disculus and Encyonopsis microcephala. A total of 73 taxa (40\%) were found exclusively in samples from carbonate substratum, and the most common (frequency > 5\%) were: Planothidium dubium, Navicula antonii, N. cariocincta, Psammothidium grischunum, Gomphonema angustatum, Rhoicosphenia abbreviata, Sellaphora pupula, Vaucheria sp., Nitzschia palea var. tenuirostris, N. fonticola, Fragilaria recapitellata, Audouinella sp., Gomphonema olivaceum, Gyrosigma acuminatum, Phaeodermatium rivulare, Platessa holsatica, Cymbopleura diminuta, Encyonopsis minuta and Phormidium tinctorium.

Results of the IndVal analysis of algal assemblages for the factor 'lithology' (Tab. 3) comprised statistically significant $(p \leq 0.01)$ indicator species with algal frequency in the samples $>10 \%$. Although many species were identified in the springs on both investigated substrata, they showed higher abundance or frequency on one substratum. A preference for ophiolitic substrata, with IndVal values $>30$, was established for: Achnanthidium minutissimum, Humidophila perpusilla, Diploneis krammeri, Encyonopsis cesatii, Gomphonema elegantissimum, Navicula radiosa, Planothidium lanceolatum and Nitzschia linearis. By contrast, a preference 
Tab. 2. The values of diversity and trophic indices of studied springs on the Mt. Konjuh with ranges of minimum (min), maximum (max), mean and standard deviation (SD), $\mathrm{t}$ - test values and $\mathrm{p}$ - statistical significance for groups of springs on carbonates (101 samples) and ophiolites (95 samples), N - number of samples per spring, S - number of taxa, J' - Pielou's index, $\mathrm{H}^{\prime}(\mathrm{ln})$ - Shannon - Wiener diversity index, TI Rott - Rott's trophic index, TID $_{\mathrm{HR}}$ - Croatian trophic index, $\mathrm{EQR}$ - ecological quality ratio with water quality ranges.

\begin{tabular}{|c|c|c|c|c|c|c|c|c|}
\hline Substrata & Spring code & $\mathrm{N}$ & $S$ & $\mathrm{H}^{\prime}$ & $\mathrm{J}^{\prime}$ & TI Rott & $\mathrm{TID}_{\mathrm{HR}}$ & EQR \\
\hline \multirow{13}{*}{ ophiolites } & $1 \mathrm{KE}$ & 12 & 48 & 1.65 & 0.63 & 2.61 & 2.23 & 0.71 (II) \\
\hline & $2 \mathrm{KE}$ & 12 & 46 & 1.50 & 0.6 & 2.14 & 2.31 & 0.67 (II) \\
\hline & $1 \mathrm{BO}$ & 9 & 55 & 1.90 & 0.67 & 2.40 & 2.41 & 0.64 (II) \\
\hline & $2 \mathrm{BO}$ & 6 & 63 & 1.93 & 0.57 & 1.77 & 2.48 & 0.61 (II) \\
\hline & $1 \mathrm{VU}$ & 9 & 63 & 2.07 & 0.65 & 2.32 & 2.49 & 0.6 (II) \\
\hline & $2 \mathrm{VU}$ & 6 & 49 & 2.01 & 0.66 & 2.29 & 2.44 & 0.62 (II) \\
\hline & MI & 5 & 56 & 2.09 & 0.7 & 2.14 & 2.28 & 0.69 (II) \\
\hline & SK & 10 & 76 & 2.22 & 0.69 & 2.32 & 2.41 & 0.64 (II) \\
\hline & KA & 9 & 54 & 1.76 & 0.62 & 2.03 & 2.30 & 0.68 (II) \\
\hline & $\min$. & & 6 & 0.32 & 0.14 & 1.39 & 1.49 & 0.49 (III) \\
\hline & $\max$. & & 47 & 2.95 & 0.91 & 3.27 & 2.77 & $1(\mathrm{I})$ \\
\hline & mean & & 19.21 & 1.79 & 0.62 & 2.23 & 2.36 & 0.65 (II) \\
\hline & SD & & 9.09 & 0.59 & 0.17 & 0.49 & 0.19 & 0.77 \\
\hline \multirow{14}{*}{ carbonates } & GB & 12 & 66 & 2.04 & 0.7 & 2.32 & 2.19 & 0.72 (II) \\
\hline & $\mathrm{KR}$ & 8 & 62 & 1.34 & 0.47 & 2.32 & 2.21 & 0.72 (II) \\
\hline & KS & 12 & 64 & 1.77 & 0.64 & 2.31 & 2.16 & 0.74 (II) \\
\hline & MV & 9 & 54 & 1.69 & 0.58 & 1.22 & 1.76 & 0.9 (I) \\
\hline & $\mathrm{PO}$ & 12 & 70 & 1.58 & 0.56 & 1.89 & 2.54 & 0.59 (III) \\
\hline & ST & 12 & 46 & 1.30 & 0.51 & 1.90 & 2.10 & 0.76 (II) \\
\hline & TA & 12 & 55 & 1.30 & 0.5 & 1.98 & 2.28 & 0.69 (II) \\
\hline & $\mathrm{TU}$ & 12 & 65 & 1.50 & 0.52 & 2.11 & 2.38 & 0.65 (II) \\
\hline & UB & 8 & 67 & 2.23 & 0.74 & 2.56 & 2.41 & 0.63 (II) \\
\hline & $\mathrm{ZP}$ & 12 & 58 & 2.23 & 0.75 & 2.19 & 2.34 & 0.66 (II) \\
\hline & $\min$. & & 5 & 0.08 & 0.03 & 0.70 & 1.05 & 0.47 (III) \\
\hline & $\max$. & & 43 & 2.83 & 0.91 & 3.18 & 2.82 & $0.96(\mathrm{I})$ \\
\hline & mean & & 19.19 & 1.74 & 0.61 & 2.06 & 2.24 & 0.7 (II) \\
\hline & SD & & 9.23 & 0.67 & 0.2 & 0.51 & 0.34 & 0.13 \\
\hline ophiolitic melange (mean) & $\mathrm{ZL}$ & 9 & 48 & 1.61 & 0.61 & 2.57 & 2.25 & 0.7 (II) \\
\hline t (ophiolites vs. carbonates) & & & -0.02 & -0.56 & -0.56 & -2.4 & 2.75 & -2.75 \\
\hline p (ophiolites vs. carbonates) & & & 0.99 & 0.57 & 0.57 & 0.02 & 0.007 & 0.007 \\
\hline
\end{tabular}

for carbonate substrata (IndVal > 30) was noted for the following species: Amphora pediculus, Odontidium mesodon, Gomphonema micropus, G. pumilum, Navicula antonii, and Planothidium dubium.

\section{Discussion}

Diatoms were the dominant algal group in the investigated springs. Our results were mostly compared with those obtained from the Alps, due to similarities in the sampling approach based on different spring types. A comparison of the presented results with previous investigations conducted in the south-eastern Alps (Cantonati 1998) revealed many species in common, such as Achnanthidium minutissimum, Odontidium (Diatoma) mesodon, Achnanthidium pyrenaicum, Cocconeis euglypta, Gomphonema pumilum s.l. and Meridion circulare. However, several species frequent in alpine springs (Brachysira brebissonii, Encyonema minutum, and Odontidium hyemalis) were either rarely present or not detected in our study. A higher similarity was found with the results of Angeli et al. (2010), who investigated diatoms in anthropogenically influenced, low altitude springs, where many eutraphentic taxa were recorded (Cocconeis, Planothidium, and Nitzschia). The highest number of taxa identified, excluding diatoms, belongs to the genera Phormidium and Chamaesiphon, which is in accordance with the results of Cantonati et al. (1996). Several species frequently recorded in this study (Chlorogloea microcystoides, Chamaesiphon polonicus, Tapinothrix varians, Audouinella sp., Vaucheria sp. and Tribonema sp.) were also found in previous investigations (e.g., Cantonati et al. 2012c). On the other hand, species frequently noted in alpine springs, such as Xenotholos kerneri, Hydrurus foetidus, Chamaesiphon starmachii, Tapinothrix janthina and Plectonema tomasinianum were not recorded in our study, presumably due to their preference for higher elevations with lower water temperature and/or siliceous substratum. For instance, a typical rheobiontic species of crenic habitats, Hydrurus foetidus, was not found in this study, possibly due to the lower water velocity of the springs investigated. Our results can be well compared with those 
KAMBEROVIĆ J., PLENKOVIĆ-MORAJ A., KRALJ BOROJEVIĆ K., GLIGORA UDOVIČ M., ŽUTINIĆ P., HAFNER D., CANTONATI M.

Tab. 3. Indicator values (IndVal) and frequency of indicative species of ophiolitic and carbonate springs on the Mt. Konjuh at $\mathrm{p}<0.01$ (4999 permutations using Monte Carlo test).

\begin{tabular}{|c|c|c|c|c|}
\hline & Taxa & IndVal & Frequency in group 1 & $\begin{array}{c}\text { Frequency in } \\
\text { group } 2 \\
\end{array}$ \\
\hline \multirow{33}{*}{ 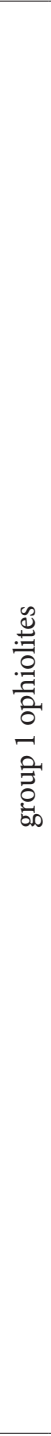 } & Achnanthidium minutissimum (Kützing) Czarnecki & 58.8 & 96 & 81 \\
\hline & Planothidium lanceolatum (Brébisson ex Kützing) Lange-Bertalot & 58.5 & 92 & 82 \\
\hline & Encyonopsis cesatii (Rabenhorst) Krammer & 50.5 & 58 & 22 \\
\hline & Humidophila perpusilla (Grunow) Lowe, Kociolek, & 46.8 & 49 & 14 \\
\hline & Nitzschia linearis (C. Agardh) W. Smith & 40 & 47 & 23 \\
\hline & Diploneis krammeri Lange-Bertalot et E. Reichardt & 38.9 & 45 & 13 \\
\hline & Navicula radiosa Kützing & 34.6 & 37 & 6 \\
\hline & Gomphonema elegantissimum E. Reichardt et Lange-Bertalot & 30.6 & 33 & 9 \\
\hline & Cymbella hantzschiana Krammer & 28.1 & 29 & 4 \\
\hline & Gomphonema parvulum (Kützing) Kützing & 23.8 & 27 & 12 \\
\hline & Gomphonema tergestinum (Grunow) Fricke & 23.8 & 27 & 4 \\
\hline & Diploneis fontanella Lange-Bertalot & 22.5 & 32 & 12 \\
\hline & Encyonopsis krammeri Reichardt & 21.1 & 26 & 11 \\
\hline & Gomphonema exilissimum (Grunow) Lange-Bertalot et E. Reichardt & 20.9 & 26 & 10 \\
\hline & Amphora lange-bertalotii var. tenuis Levkov et Metzeltin & 20.4 & 22 & 3 \\
\hline & Navicula leistikowii Lange-Bertalot & 20.2 & 24 & 7 \\
\hline & Encyonopsis fonticola (Hustedt) Krammer & 19.3 & 21 & 4 \\
\hline & Cymbella parva (W.Smith) Kirchner & 17.5 & 21 & 7 \\
\hline & Achnanthidium exile (Kützing) Heiberg & 16.8 & 19 & 3 \\
\hline & Navicula cataracta-rheni Lange-Bertalot & 15.4 & 15 & 0 \\
\hline & Tryblionella angustata W. Smith & 15.1 & 18 & 6 \\
\hline & Chlorogloea microcystoides Geitler & 14.6 & 17 & 5 \\
\hline & Pseudanabaena sp. Lauterborn & 13.6 & 14 & 1 \\
\hline & Nitzschia amphibia Grunow & 13.3 & 14 & 3 \\
\hline & Surirella terricola Lange-Bertalot et E. Alles & 12.6 & 14 & 5 \\
\hline & Eunotia soleirolii (Kützing) Rabenhorst & 11.5 & 12 & 0 \\
\hline & Rhopalodia parallela (Grunow) O. Müller & 10.7 & 12 & 1 \\
\hline & Encyonema silesiacum (Bleisch) D.G.Mann & 10.1 & 12 & 2 \\
\hline & Diploneis elliptica (Kützing) Cleve & 9.9 & 10 & 1 \\
\hline & Eucocconeis laevis (Østrup) Lange-Bertalot & 9.6 & 10 & 3 \\
\hline & Nitzschia capitellata Hustedt & 9.2 & 12 & 2 \\
\hline & Tryblionella angustatula (Lange-Bertalot) Cantonati et Lange-Bertalot & 9.0 & 9 & 0 \\
\hline & Hantzschia amphioxys (Ehrenberg) Grunow & 8.3 & 9 & 1 \\
\hline \multirow{20}{*}{ 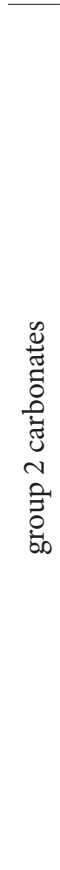 } & Amphora pediculus (Kützing) Grunow ex A. Schmidt & 56.1 & 50 & 83 \\
\hline & Odontidium mesodon (Ehrenberg) Kützing & 44.3 & 18 & 50 \\
\hline & Navicula antonii Lange-Bertalot & 39.4 & 0 & 39 \\
\hline & Planothidium dubium (Grunow) Round et Bukhtiyarova & 39.4 & 0 & 39 \\
\hline & Gomphonema micropus Kützing & 34.7 & 29 & 52 \\
\hline & Gomphonema pumilum (Geitler) Bohunická et J. R. Johansen & 31.2 & 12 & 35 \\
\hline & Achnanthidium pyrenaicum (Hustedt) H. Kobayasi & 29.1 & 21 & 38 \\
\hline & Navicula tripunctata (O. F. Müller) Bory de Saint-Vincent & 26.1 & 3 & 29 \\
\hline & Psammothidium grischunum (Wuthrich) L. Bukhtiyarova et Round & 23.9 & 0 & 24 \\
\hline & Nitzschia oligotraphenta (Lange-Bertalot) Lange-Bertalot & 22.5 & 4 & 24 \\
\hline & Achnanthidium affine (Grunow) Czarnecki & 20.0 & 5 & 22 \\
\hline & Gomphonema angustatum (Kützing) Rabenhorst & 18.3 & 0 & 18 \\
\hline & Nitzschia fonticola (Grunow) Grunow & 18.3 & 0 & 18 \\
\hline & Navicula cryptotenelloides Lange-Bertalot & 18.0 & 4 & 19 \\
\hline & Rhoicosphenia abbreviata (C.Agardh) Lange-Bertalot & 16.5 & 0 & 17 \\
\hline & Sellaphora pupula (Kützing) Mereschkovsky & 16.5 & 0 & 17 \\
\hline & Vaucheria sp. A. P. de Candolle & 15.6 & 0 & 16 \\
\hline & Phormidium retzii Kützing ex Gomont & 14.9 & 3 & 16 \\
\hline & Nitzschia palea var. tenuirostris Grunow & 11.0 & 0 & 11 \\
\hline & Fragilaria recapitellata $\mathrm{H}$. Lange-Bertalot et D. Metzeltin & 10.1 & 0 & 10 \\
\hline
\end{tabular}


from 16 springs and streams of the Dolomiti Bellunesi National Park in Northern Italy (Cantonati 2008), where a total of 65 algal taxa apart from diatoms, and 1-12 taxa per spring location were recorded. The exceptional heterogeneity of springs, resulting in richness of rare and endangered species already highlighted in several studies (Aboal et al. 1998, Werum and Lange-Bertalot 2004, Cantonati et al. 2006) was confirmed for the area investigated. Overall, one third of all taxa identified in the present study (36.3\%) was noted in one spring only, which is slightly lower than $40 \%$ recorded by Cantonati and Spitale (2009) and clearly less than 58\% noted by Bertrand et al. (2004). All of the aforementioned studies refer to springs as unique habitats, each requiring a specific diversity estimation.

Hierarchical clustering based on relative algal abundance clustered springs into six groups. These results were in partial agreement with Cantonati et al. (2012b, c), whose spring classification included 6 diatom-based and 7 groups based on algal taxa except diatoms. Species typical for rheocrenes on carbonates, such as Achnanthidium pyrenaicum and Nitzschia fonticola (Cantonati et al. 2012b), have been detected with high abundance in the spring MV. Groups of rheocrenes on carbonates ( $3^{\text {rd }}$ and $5^{\text {th }}$ cluster) showed greatest similarity with rheocrenes on carbonate substrata with $\mathrm{NO}_{3}{ }^{-}$enrichment or shading described by Cantonati et al. (2012b). Diatom species shared by Alpine and Dinaric carbonate springs were Odontidium mesodon, Achnanthidium pyrenaicum, Amphora pediculus, Meridion circulare, Cocconeis lineata, C. pseudolineata, and Sellaphora nigri. This congruence was expected, since the measured increased nitrate concentrations in these springs determined a higher number of eutraphentic species (e.g., Amphora pediculus). The cyanoprokaryote Tapinothrix varians and the red alga Audouinella spp. showed affinity for shaded springs, as detected in some earlier studies (Cantonati et al. 1996, Cantonati et al. 2012c). However, certain differences were observed when the springs on ophiolites were compared with springs emerging from siliceous aquifers. In a comparison of algal assemblages, the highest resemblance was observed between rheocrenes on ophiolites and group 4 (rheocrenes on carbonate substrata with lower conductivity or seasonal desiccation) by Cantonati et al. (2012b). Species in common were Planothidium frequentissimum, Achnanthidium dolomiticum, Meridion circulare and several indicators of desiccation, e.g. Humidophila perpusilla and $H$. contenta. Moreover, two new records for the diatom flora of Bosnia and Herzegovina were found in the investigated ophiolite springs: Achnanthidium dolomiticum, a species previously recorded in springs on dolomite substrata (Cantonati and Lange-Bertalot 2006), and Cymbella tridentina, a rheophilic species of carbonate headwaters (Cantonati et al. 2010). A common feature of dolomite and ophiolite springs is a relatively high magnesium concentration, presumably a key determinant for the distribution of A. dolomiticum. The largest group, rheohelocrenic springs on ophiolites, was compatible with hygropetric rheocrenes on carbonate substrata in Cantonati et al. (2012b), with several species in common: Denticula tenuis, Encyonopsis ce- satii, and E. microcephala. Despite different lithology, springs on ophiolites and springs on carbonate substrata had a very similar algal composition. The greatest concurrence in algal communities recorded in this study with springs on siliceous substrata from previous studies was noted for the sole spring on ophiolitic melange (ZL), with several species of Gomphonema and Eunotia in common.

The influence of lithology on algal assemblages has been emphasized by several authors (Cantonati 1998, Werum 2001, Cantonati et al. 2006, Wojtal 2013), typically considering springs on different, carbonate and siliceous, lithologies. Moreover, phycological studies focusing on springs on ophiolites (silicate rocks) were very rare. When algal assemblages of springs on peridotite and serpentinite (ophiolites) from this study were compared with springs on amphibolite in Germany (Werum 2001), a high similarity in species composition was noted. However, many species commonly occurring on ophiolites in this study, such as A. minutissimum, Humidophila perpusilla, Diploneis fontanella, Nitzschia linearis and Planothidium lanceolatum, were not good indicators of aquifer lithology because of their widespread distribution (Werum 2001). On the contrary, species that had lower abundance and higher frequency, as detected by IndVal analysis, proved to be more reliable indicators of an aquifer's lithology. The IndVal analysis singled out the following species as having a preference for ophiolites: Amphora langebertalotii var. tenuis, Cymbella hantzschiana, C. parva, Encyonopsis cesatii, E. krammeri, Gomphonema parvulum, G. tergestinum and Navicula leistikowii. Some of the species listed are typical for calcareous fens, such as E. cesatii (Fránková et al. 2009), others are widespread such as G. parvulum (Abarca et al. 2014).

Results of the indicator-species analysis for carbonate substrata were in accordance with previous studies (Cantonati et al. 2012b, c), in which several rheophilic species (Achnanthidium pyrenaicum, Odontidium mesodon, Gomphonema micropus, G. pumilum, Planothidium dubium) or species with a preference for nitrogen enrichment $(\mathrm{Na}$ vicula tripunctata and Amphora pediculus) were noted. A low resemblance has been found when our results on ophiolite-spring algal assemblages were compared with results from previous investigations in springs emerging from other types of siliceous substrata such as granite and rhyolite (Werum 2001, Kapetanović and Hafner 2007). Species typical for rheocrenes and helocrenes on siliceous substrata of the south-eastern Alps (Cantonati et al. 2012c) or detected in the Pyrenees (Sabater and Roca 1992), were not found in the ophiolite springs studied. In the latter, genera typical for siliceous substrata, such as Tabellaria, Eunotia and Pinnular$i a$, were either missing or occurring only with a few species. Nevertheless, a large number of shared species (110) suggests fewer differences in the structure of algal communities between carbonates and ophiolites (this study) than between ultramaphites (this study) and other types of siliceous substrata (e.g. granite, rhyolite; algal community composition data obtained from the literature). In addition, a species typical for carbonate substrata, Tapinothrix varians (Cantonati 
et al. 1996), was found in all springs regardless of lithological substratum, and especially in springs with higher current velocity. On the other hand, Tapinothrix janthina, the vicariant species typical for springs on silicates, was not found in the present study. Our results suggest that algal assemblages in springs emerging from ophiolites and springs emerging from other types of siliceous substrata should be separately analyzed in future studies.

The total number of species in springs can greatly depend on the lithology or the heterogeneity of microhabitats. Nascimbene et al. (2011) recorded higher numbers of diatoms, plants and lichens from springs on siliceous substrata, whilst other algal groups (including cyanoprokaryotes) were more numerous on carbonate substrata. On the other side, Frankovà et al. (2009) pointed out that the number of diatom species decreased significantly from mineral-rich to mineral-poor spring fen sites. Although a higher number of taxa was noted in springs on carbonates than in those on ophiolites, differences between averages of diversity indices were not significant. The total number of diatom taxa found in the 20 springs of Mt. Konjuh (187) was higher than in 17 Swiss springs (118 taxa; Taxböck and Preisig 2007), very similar to Pyrenean springs (194 taxa; Sabater and Roca 1992), and lower than in 30 springs in south-eastern Alps (250 taxa; Cantonati 1998). Although the average value of the Shannon-Wiener Diversity Index indicated a moderately high diversity of algal species $\left(\mathrm{H}^{\prime}=1.75\right)$, it was lower than the value obtained for Alpine springs $\left(\mathrm{H}^{\prime}=2\right.$, Cantonati 1998). Several authors emphasized a relationship between lower altitudes and higher nitrate concentrations in springs (Aboal et al. 1998, Cantonati and Spitale 2009), which can result in a decrease of diatom species (Bertrand et al. 2004). A wide range in the total number of species per individual sample (from 5 to 47 ) and a high number of taxa per spring location (from 46 to 76 ) indicate the need to sample different microhabitats to achieve a more accurate biodiversity assessments of spring habitats.

The algal assemblages in wetland habitats around springs and streams of the Vranica Mountain, Bosnia and Herzegovina (Kapetanović and Hafner 2007), especially those on siliceous substrata, differ from those of the springs studied on Mt. Konjuh by the preponderance of acidophilous taxa (Brachysira brebissonii, Eunotia incisa, Tabellaria flocculo$s a$, and many species from the genera Neidium and Eunotia). Acidophilous species were rarely found on Mt. Konjuh, due to ultramafic and calcareous lithologies resulting in higher $\mathrm{pH}$ values. On the other hand, many eutraphentic species were noted (32.7\%). This proportion was higher than that recorded by Cantonati and Spitale (2009) in the Dolomiti Bellunesi National Park in Italy (22\%). Altough natural diatom distribution could be altered by antrhopogenic influences, Wojtal (2013) pointed out that springs with high nutrient concentrations also show heterogeneity of diatom assemblage composition indicating the great potential of springs for biodiversity assessment. Angeli et al. (2010) pointed out that any morphological alteration of springs leads to great disturbance of their diatom communities, which was also the case in many springs studied herein. Phormidium retzii, an indicator of nitrogen enrichment (Cantonati et al. 2012c), was found in seven springs on Mt. Konjuh, indicating anthropogenic impact and a deteriorated ecological status.

Values of Rott's TI were high due to the dominance of highly competitive meso- and eutraphentic algal species, which is compatible with the results of some previous investigations of low-altitude springs in Germany (Werum 2001), and anthropogenically impacted springs in Austria (Gesierich and Kofler 2010), thus emphasizing that an increase in nitrate leads to decreasing numbers of sensitive and rare species. The mean values of the Rott TI (2.06 and 2.23 in carbonate and ophiolitic springs, respectively) belong in the same trophic class as a half of the investigated springs from the River Adige basin, and are higher than Rott's TI from springs of Trentino (Angeli et al. 2010). Adversely, the $\mathrm{EQR}$ and TID $_{\mathrm{HR}}$ values indicated for one level lower trophic class than Rott's TI in the present investigation, presumably due to geographical adaptation of the species indicator values. However, the use of indices developed for rivers in the trophic-status assessments of springs could undergo certain limitations, in particular in the case of spring types markedly differing from streams.

\section{Conclusion}

The study provides the first information on the biodiversity of algal communities in the mountain springs of north-eastern Bosnia and Herzegovina. Although springs had long been neglected in hydrobiological investigations, recent studies emphasize the importance of these habitats in biodiversity conservation. Despite the visible anthropogenic impacts in springs of Mt. Konjuh, a moderately high diversity of algal species per spring location was detected. Results of this study revealed that springs on ophiolites should be analyzed separately from springs on other siliceous substrata in future studies dealing with community structure in different spring types. Due to the high heterogeneity of spring habitats, in order to preserve spring species diversity of Mt. Konjuh the conservation of springs as a group of habitats is required, and not only the protection of individual spring sites. Special focus should be directed towards preserving small rheohelocrenic springs, which are usually ignored in management and protection plans, even though they have a great potential in species conservation.

\section{Acknowledgements}

We are grateful to Elvir Babajić for help in the identification of the lithology of substrata, to Selvir Kamberović for help in data processing, and the Federal Ministry of Education and Science in Bosnia and Herzegovina for providing funding for a research visit to the MUSE - Museo delle Scienze, Trento, Italy. 


\section{References}

Abarca, N., Jahn, R., Zimmermann, J., Enke, N., 2014: Does the cosmopolitan diatom Gomphonema parvulum (Kutzing) Kutzing Have a Biogeography? PLoS One 9(1), e86885

Aboal, M., Puig, M.A., Prefasi, M., 1998: Diatom assemblages in springs in Castellơn province, Eastern Spain. Algological Studies 90, 79-95.

Alexander, E. B., Coleman, R.G., Keeler Wolf, T., Harrison, S.P., 2007: Serpentine geoecology of western North America: geology, soils, and vegetation. Oxford University Press, New York.

APHA, AWWA, and WEF, 1999: Standard methods for the examination of water and wastewater $20^{\text {th }}$ edition, Washington DC.

Angeli, N., Cantonati, M., Spitale, D., Lange-Bertalot, H., 2010: A comparison between diatom assemblages in two groups of carbonate, lowland springs with different levels of anthropogenic disturbances. Fottea 10, 115-128.

Anonymous, 2013: Official Gazzete of the Republic of Croatia $73 / 13,151 / 14$ and 78/15 (in Croatian).

Anonymous, 2016: Methods of sampling, laboratory analysis and determination of the ecological quality ratio of the biological quality elements. Retrieved on $22^{\text {nd }}$ February 2017 from http:// www.voda.hr/hr/metodologije.

Babajić, E., 2009: Petrological-geochemical and geotectonic characteristics of mafite magmatic rocks of the Krivaja-Konjuh ophiolite complex. Doctoral thesis. Faculty of Mining, Geology and Civil Engineering. University of Tuzla. Tuzla (In Bosnian).

Beck, G., 1928: Zur Kenntnis der Algen von Bosnien. Bulletin De L'Institute et du Jardin Botaniques de 1'Université de Beograd 1, 36-42.

Bertrand, J., Renon, J. P., Monnier O., 2004: Relation “diatomées épiphytes-bryophytes" dans les tourbières du Mont Lozère (France). Vie et Milieu 54, 59-70.

Blagojević, S., 1974: The development of periphyton in open devices waterworks on karstic springs. Doctoral thesis. Faculty of Science, University of Zagreb (In Croatian).

Blagojević, S., 1976: Contribution on the Cyanophyceae of karst springs. Acta Botanica Croatica 35, 207-215.

Blagojević, S., 1979: Contribution on the algae of karst springs: Chlorophyceae. Acta Botanica Croatica 38, 125-132.

Cantonati, M., 1998: Diatom communities of springs in the Southern Alps. Diatom Research 13, 201-220.

Cantonati, M., 2008: Cyanoprokaryotes and algae other than diatoms in springs and streams of the Dolomiti Bellunesi National Park (Northern Italy). Algological Studies 126, 113-136.

Cantonati, M., Füreder L., Gerecke, R., Jüttner, I., Cox, E.J., 2012a: Crenic habitats, hotspots for freshwater biodiversity conservation: toward an understanding of their ecology. Freshwater Science 31, 463-480.

Cantonati, M., Angeli, N., Bertuzzi, E., Spitale, D., 2012b: Diatoms in springs of the Alps: spring types, environmental determinants, and substratum. Freshwater Science 31, 499-524.

Cantonati, M., Rott, E., Spitale, D., Angeli, N., Jiří, K., 2012c: Are benthic algae related to spring types? Freshwater Science 31, 481-498.

Cantonati, M., Gerecke, R., Bertuzzi, E., 2006: Springs of the Alps - sensitive ecosystems to environmental change: from biodiversity assessments to long-term studies. Hydrobiologia 562, 59-96.

Cantonati, M., Lange-Bertalot, H. Scalfi, A., Angeli, N., 2010: Cymbella tridentina sp. nov. (Bacillariophyta), a crenophilous diatom from carbonate springs of the Alps. Journal of the North American Benthological Society 29, 775-788.

Cantonati, M., Lange-Bertalot, H., 2006: Achnanthidium dolomiticum sp. nov. (Bacillariophyta) from oligotrophic mountain springs and lakes fed by dolomite aquifers. Journal of Phycology $42,1184-1188$.

Cantonati, M., Lange-Bertalot, H., 2009: Geissleria gereckei sp. nov. (Bacillariophyta) from leaf-litter covered stones of very shaded carbonate mountain springs with extremely low discharge. Phycological Research 57, 171-177.

Cantonati, M., Lange-Bertalot, H., 2010: Diatom biodiversity of springs in the Berchtesgaden National Park (northern Alps, Germany), with the ecological and morphological characterization of two species new to science. Diatom Research 25, 251-280.

Cantonati, M., Lange-Bertalot, H., 2011: Diatom monitors of closeto-pristine, very-low alkalinity habitats: three new Eunotia species from springs in Nature Parks of the south-eastern Alps. Journal of Limnology 70, 209-221.

Cantonati, M., Rott, E., Pipp, E., 1996: Ecology of cyanophytes in mountain springs of the River Sarca catchment (AdamelloBrenta Regional Park, Trentino, Northern Italy). Algological Studies 83, 145-162.

Cantonati, M., Spitale, D., 2009: The role of environmental variables in structuring epiphytic and epilithic diatom assemblages in springs and streams of the Dolomity Bellunesi National Park (south-eastern Alps). Fundamental and Applied Limnology 174, 117-133.

Clarke, K.R., Warwick, R.M., 2001: Change in marine communities: an approach to statistical analysis and interpretation, $2^{\text {nd }}$ edition. PRIMER-E, Plymouth, 172.

Coleman, R.G., Jove, C., 1992: Geological origin of serpentinites. In: Baker, A. J. M., Proctor, J., Reeves, R. D. (eds.), The vegetation of ultramafic (serpentine) soils, 1-18. Intercept, Andover.

Cvijan, M., Blaženčić, J., 1996: Flora of the algae of Serbia. Cyanophyta 1. Naučna knjiga, Beograd (in Serbian).

Dedić, A., Plenković-Moraj A., Kralj BorojevićK., Hafner D., 2015: The first report on periphytic diatoms on artificial and natural substrate in the karstic spring Bunica, Bosnia and Herzegovina. Acta Botanica Croatica 74, 393-406.

Dilek, Y., Furnes, H., 2011: Ophiolite genesis and global tectonics: Geochemical and tectonic fingerprinting of ancient oceanic lithosphere. Geological society of America Bulletin 123, 387-411.

Dufrene, M., Legendre, P., 1997: Species assemblages and indicator species: the need for a flexible asymmetrical approach. Ecological Monographs 67, 345-366.

Falasco, E., Bona, F., 2011: Diatom community biodiversity in an Alpine protected area: a study in the Maritime Alps Natural Park. Journal of Limnology 70, 157-167.

Fránková, M., Bojková, J., Poulíčková A., Hájek. M., 2009: The structure and species richness of the diatom assemblages of the western Carpathian spring fens along the gradient of mineral richness. Fottea 9, 355-368.

Gesierich, D., Kofler, W., 2010: Epilithic diatoms from rheocrene springs in the eastern Alps (Vorarlberg, Austria). Diatom Research 25, 43-66.

Grandin, U., 2006: PC-ORD version 5: A user-friendly toolbox for ecologists. Journal of Vegetation Science 17, 843-844.

Gutwinski, R., 1899: Ein Beitrag zur Kenntniss der fossilen Diatomaceen Bosniens. Wissenschaftliche Mittheilungen aus Bosnien und Hercegowina 6, 679-684.

Hafner, D., 2009: Diatom of the karstic springs in Herzegovina. Radovi hrvatskog društva za znanost i umjetnost, 11, 209-220 (In Croatian).

Hofmann, G., Werum, M., Lange-Bertalot, H., 2011: Diatomeen im Süßwasser - Benthos von Mitteleuropa. Bestimmungsflora Kieselalgen für die ökologische Praxis. Über 700 der häufigsten Arten und ihre Ökologie. Ganter, Rugell. 
Hustedt, F., 1930: Die Süßwasser flora Mitteleuropas. Heft 10: Baccillariophyta (Diatomeae) $2^{\text {nd }}$ edition. Jena.

Hustedt, F., 1945: Diatomeen aus Seen und Quellgebieten der Balkan-Halbinsel. Archiv für Hydrobiologie 40, 867-973.

Institute for standardization of Bosnia and Herzegovina, 2000: Water quality - determination of nitrite - molecular absorption spectrometric method (BAS EN 26777:2000). Retrieved from http://www.bas.gov.ba/standard/?natstandard_document_ id=239905.

Institute for standardization of Bosnia and Herzegovina, 2002a: Water quality-determination of ammonium - Part 1: Manual spectrometric method (BAS ISO 7150-1: 2002). Retrieved from http://www.bas.gov.ba/standard/?natstandard_document_id=241854.

Institute for standardization of Bosnia and Herzegovina, 2002b: Water quality - determination of nitrate - Part 3: Spectrometric method using sulfosalicylic acid (BAS ISO 7890-3:2002). Retrieved from http://www.bas.gov.ba/standard/?natstandard_ document_id $=241858$.

John, D. M., Whitton, B. A., Brook, A. J., 2002: The freshwater algal flora of the British Isles: an identification guide to freshwater and terrestrial algae. Cambridge University Press, Cambridge.

Kapetanović, T., Hafner, D., 2007: Diatoms of wet habitats in the subalpine belt of Mt. Vranica (Bosnia and Herzegovina). In: Kusber, W. H., Jahn, R. (eds.), Proceedings of the $1^{\text {st }}$ Central European Diatom Meeting 2007 Berlin-Dahlem. BGBM Press, Berlin, 73-78.

Kelly, M. G., Cazaubon, A., Coring, E., Dell'Uomo, A., Ector, L., Goldsmith, B., Guasch, H., Hürlimann, J., Jarlman, A., Kawecka, B., Kwandrans, J., Laugaste, R., Lindstrøm, E.A., Leitao, M., Marvan, P., Padisák, J., Pipp, E., Prygiel, J., Rott, E., Sabater, S., van Dam, H., Vizinet, J., 1998: Recommendations for the routine sampling of diatoms for water quality assessments in Europe. Journal of Applied Phycology 10, 215-224.

Kelly, M.G., Whitton, B. A., 1995: The trophic diatom index: a new index for monitoring eutrophication in rivers. Journal of Applied Phycology 7, 433-444.

Komárek, J., Anagnostidis, K., 1998: Cyanoprokaryota 1. Teil: Chroococcales. In: Ettl H., Gärtner, G., Heynig, H., Mollenhauer, D. (eds.), Süsswasserflora von Mitteleuropa 19/1, Gustav Fischer, Jena-Stuttgart-Lübeck-Ulm.

Komárek, J., Anagnostidis, K., 2005: Cyanoprokaryota 2. Teil/ 2nd Part: Oscillatoriales. In: Büdel, B., Krienitz, L., Gärtner, G., Schagerl, M. (eds.), Süsswasserflora von Mitteleuropa 19/2, Elsevier/Spektrum, Heidelberg.

Krammer, K., 2000: The genus Pinnularia. In: Lange-Bertalot, H., (ed.), Diatoms of Europe, Volume 1. A.R.G. Gantner Verlag K. G. 1-703.

Krammer, K., 2002: Cymbella. In: Lange-Bertalot, H., (ed.), Diatoms of Europe, Volume 3. A.R.G. Gantner Verlag K.G. 1-584.

Krammer, K., H. Lange-Bertalot, H., 1988. Bacillariophyceae. 2. Teil: Bacillariaceae, Epithemiaceae, Surirellaceae. In Ettl, H., Gerloff, J., Heynig, H., Mollenhauer, D. (eds.), Süsswasserflora von Mitteleuropa. Vol. 2/2. Gustav Fischer Verlag, Jena, 1-596.

Krammer, K., Lange-Bertalot, H. 1986: Bacillariophyceae 1. Teil: Naviculaceae. In: Ettl, H., Gerloff, J., Heynig, H., Mollenhauer, D. (eds.), Süsswasserflora von Mitteleuropa, 2/1. Gustav Fischer Verlag, Stuttgart, New York, 1-876.

Krammer, K., Lange-Bertalot, H. 2000: Bacillariophyceae 3. Teil: Centrales, Fragilariaceae, Eunotiaceae. In: Ettl, H., Gerloff, J., Heynig, H., Mollenhauer, D. (eds.), Süßwasserflora von Mitteleuropa Band 2/3 (2nd edition), Spektrum Akademischer Verlag, Heidelberg, 1-599.

Krammer, K., Lange-Bertalot, H. 2004a: Bacillariophyceae. 3. Teil: Centrales, Fragilariaceae, Eunotiaceae. In: Ettl, H., Gerloff, J.,
Heynig, H., Mollenhauer, D. (eds.), Süsswasserflora von Mitteleuropa 2/3. Spektrum Akademischer Verlag, Heidelberg, Berlin, 1-598.

Krammer, K., Lange-Bertalot, H., 2004b: Bacillariophyceae 4. Teil: Achnanthaceae. Kritische Ergänzungen zu Navicula (Lineolatae) und Gomphonema Gesamtliteraturverzeichnis Teil 1- 4. In: Ettl, H., Gärtner, G., Gerloff, J., Heynig, H., D. Mollenhauer (eds.), Süsswasserflora von Mitteleuropa. Vol. 2/4. Spektrum Akademischer Verlag, Heidelberg, Berlin, 1-468.

Krebs, C. J., 1999: Ecological methodology, $2^{\text {nd }}$ ed. Addison-Welsey Educational Publishers, Inc., Menlo Park, CA.

Kudumović Dostović, F., 2012: Regional-geographical characteristics of north-eastern Bosnia. Doctoral thesis. Faculty of Natural Sciences and Mathematics. University of Tuzla. Tuzla (In Bosnian).

Lange-Bertalot, H., Hofmann, G., Werum, M., Cantonati, M., 2017: Freshwater benthic diatoms of central Europe: over 800 common species used in ecological assessment. Cantonati, M., Kelly, M.G., Lange-Bertalot, H. (eds.), English edition with updated taxonomy and added species. Koeltz Botanical Books, $1-942$.

Legendre, P., Legendre, L., 1998: Numerical ecology. $2^{\text {nd }}$ english edition. Elsevier, Amsterdam.

Lugović, B., Atherr, R., Raczhek, I., Hofmann, A.W., Majer, M., 1991: Geochemistry of peridotites and mafic igneous rocks from the Central Ophiolite Belt, Yugoslavia. Contribution to Mineralogy and Petrology 106, 201-216.

Nascimbene, J., Spitale, D., Thüs, H., Cantonati, M., 2011: Congruencies between photoautotrophic groups in springs of the Italian Alps: implications for conservation strategies. Journal of Limnology 70, 3-8.

Operta, M., 2017: Contribution to knowledge of contains on peridotite rocks of the Krivaja-Konjuh ophiolitic complex (Massif). Bulletin of the Chemists and Technologists of Bosnia and Herzegovina 48, 27-34.

Poulíčková, A., Hašler, P., Kitner, M., 2005: Cyanobacteria and algae. In: Poulíčková, A., Hájek, M., Rybníček, K. (eds.), Ecology and palaeoecology of spring fens of the West Carpathians (Monography), 105-130. Palacký University, Olomouc.

Protić, Đ., 1897: Contribution to knowledge of algal flora of Bosnia and Herzegovina, peculiarly areas of Sarajevo, Vareš and Mostarsko blato, excluding diatoms. Glasnik Zemaljskog Muzeja Bosne i Hercegovine 9, 539-559 (In Bosnian).

Protić, Đ., 1899: Beitrag zur Kenntniss der Kieselalgen Bosniens und der Hercegovina Wissenschaftliche Mittheilungen aus Bosnien und Hercegowina 6, 708-718.

Protić, Đ., 1901: Third contribution to knowledge of algal flora of Bosnia and Herzegovina. Glasnik Zemaljskog Muzeja Bosne i Hercegovine 13, 201-226 (In Bosnian).

Protić, Đ., 1908: Fourth contribution to knowledge of algal flora of Bosnia and Herzegovina. Glasnik Zemaljskog Muzeja Bosne i Hercegovine 19, 191-202 (In Bosnian).

Ristić, P., Panić, R., Mudrinić, Č., Likić, J., 1967: Magmatism and geohemistry of Mountain Konjuh. Arhiv za tehnologiju 3-4, 3-17 (In Bosnian).

Rott, E., Pipp, E., Pfister, P., van Dam, H., Ortler, K., Binder, N., 1999: Indikationslisten für Aufwuchsalgen in österreichischen Fliessgewässern. Teil 2: Trophieindikation. Bundesministerium für Land-und Forstwirtschaft, Wien.

Sabater, S., Roca, J. R., 1990: Some factors affecting distribution of diatom assemblages in Pyrenean springs. Freshwater Biology 24, 493-507.

Sabater, S., Roca, J. R., 1992: Ecological and biogeographical aspects of diatom distribution in Pyrenean springs. British Phycological Journal 7, 203-213. 
Shannon, C. E., Weaver, W., 1949: The Mathematical theory of Communication. University of Illionis Press, Urbana, 125.

Spitale, D., 2007: Assessing the ecomorphology of mountain springs: suggestion from a survey in the South - Eastern Alps. In: Cantonati, M., Bertuzzi, E., Spitale, D. (eds.), The spring habitat: Biota and sampling methods. Monografie del Museo Tridentino di Scienze Naturali 4. Museo Tridentino di Scienze Naturali, Trento, 31-44.

Starmach, K., 1985: Chrysophyceae und Haptophyceae. In: Ettl H., Gärtner, G., Heynig, H., Mollenhauer, D. (eds.), Süsswasserflora von Mitteleuropa. Band 1. Gustav Fischer Verlag, Stuttgart.1-515.

Stevenson, R.J., Bahls, L. L., 1999: Periphyton protocols. In: Barbour, M.T., Gerritsen, J., Snyder, B. D., Stribling, J. B. (eds.), Rapid bioassessment protocols for use in streams and wadeable rivers: periphyton, benthic macroinvertebrates and fish, $2^{\text {nd }}$ ed., Report EPA 841-B-99-002. U. S. Environmental Protection Agency, Office of Water, Washington, DC.

Taxböck, L., Preisig, H. R., 2007: The diatom communities in Swiss springs: a first approach. Proceedings of the $1^{\text {st }}$ Central European Diatom Meeting. In: Kusber, W. H., Jahn, R. (eds.), Botanic Garden and Botanical Museum Berlin-Dahlem. Freie Universität Berlin, Berlin, 163-168.
Taxböck L., Linder H. P., Cantonati M. 2017: To what extent are Swiss springs refugial habitats for sensitive and endangered diatom taxa? Water 9, 967.

Trubelja, F., Marchig, V., Burgath, K.P., Vujović, Ž., 1995: Origin of the jurassic tethyan ophiolites in Bosnia: A geochemical approach to tectonic setting. Geologia Croatica 48, 49-66.

van Dam, H., Mertens, A., Sinkeldam, J., 1994: A coded checklist and ecological indicator values of freshwater diatoms from the Netherlands. Netherlands Journal of Aquatic Ecology 28, 117-133.

Werum, M., 2001: Die Kieselalgengesellschaften in Quellen. Abhängigkeit von Geologie kostenlos und anthropogener Beeinfl ussung in Hessen (Bundesrepublik Deutschland). Wiesbaden.

Werum, M., Lange-Bertalot, H., 2004: Diatoms in springs from Central Europe and elsewhere under influence of hydrologeology and anthropogenic impacts. In: Lange-Bertalot, $\mathrm{H}$. (ed.), Iconographia Diatomologica 13. A. R. G. Gantner Verlag K. G., Ruggell, 1-479.

Wojtal, Z. A., 2013: Species composition and distribution of diatom assemblages in spring waters from various geological formations in southern Poland. Bibliotheca diatomologica $59,1-436$. 